NBI-HE-94-31

gr-qc/9406044

June 1994

\title{
Fundamental Constants and the Problem of Time
}

\author{
A. Carlini $\diamond$ 母 and J. Greensite* ${ }^{*}$ \\ NORDITA $\diamond$ and The Niels Bohr Institute * \\ Blegdamsvej 17 \\ DK-2100 Copenhagen Ø, Denmark
}

\begin{abstract}
We point out that for a large class of parametrized theories, there is a constant in the constrained Hamiltonian which drops out of the classical equations of motion in configuration space. Examples include the mass of a relativistic particle in free fall, the tension of the Nambu string, and Newton's constant for the case of pure gravity uncoupled to matter or other fields. In the general case, the classically irrelevant constant is proportional to the ratio of the kinetic and potential terms in the Hamiltonian. It is shown that this ratio can be reinterpreted as an unconstrained Hamiltonian, which generates the usual classical equations of motion. At the quantum level, this immediately suggests a resolution of the "problem of time" in quantum gravity. We then make contact with a recently proposed transfer matrix formulation of quantum gravity and discuss the semiclassical limit. In this formulation, it is argued that a physical state can obey a (generalized) Poincaré algebra of constraints, and still be an approximate eigenstate of 3-geometry. Solutions of the quantum evolution equations for certain minisuperspace examples are presented. An implication of our proposal is the existence of a small, inherent uncertainty in the phenomenological value of Planck's constant.
\end{abstract}

\footnotetext{
${ }^{1}$ Email: carlini@nbivax.nbi.dk

${ }^{2}$ Permanent address: Physics and Astronomy Dept., San Francisco State University, San Francisco CA 94720. Email: greensit@stars.sfsu.edu
} 


\section{Introduction}

Actions which are invariant under reparametrizations of the time variable typically have Hamiltonian constraints of the form $H[p, q]=0$, which can be viewed, in an initial value problem, as constraining the initial state $\{p, q\}_{0}$. In the Dirac quantization scheme, these constraints on the conjugate variables become constraints on the physical states, so that instead of the usual Schrodinger evolution equation one has

$$
H\left[-i \frac{\partial}{\partial q^{a}}, q^{a}\right] \Psi\left[q^{a}\right]=0
$$

In cases where the constraints are parabolic, such as the parametrized non-relativistic particle, or parametrized scalar field theory, the constraint equation (II) can often be treated exactly as a Schrodinger equation; the coordinate whose derivatives appear only to first order is identified with time (in the non-relativistic case), or "many-fingered time" (in the scalar field-theory case) [1]. On the other hand, for hyperbolic constraints such as the Klein-Gordon equation with an arbitrary background metric $g_{\mu \nu}$

$$
\left[-g^{\mu \nu} \frac{\partial^{2}}{\partial x^{\mu} \partial x^{\nu}}+m^{2}\right] \psi\left(x^{\alpha}\right)=0
$$

or the Wheeler-de Witt equation

$$
\begin{aligned}
& \left\{-\kappa^{2} G_{i j k l} \frac{\delta^{2}}{\delta g_{i j} \delta g_{k l}}+\frac{1}{\kappa^{2}} \sqrt{g}\left(-{ }^{3} R\right)\right\} \Phi=0 \\
& G_{i j k l}=\frac{1}{2 \sqrt{g}}\left[g_{i k} g_{j l}+g_{i l} g_{j k}-g_{i j} g_{k l}\right]
\end{aligned}
$$

it has proven difficult to identify an appropriate evolution parameter and a unique, positive, and conserved probability measure. In quantum gravity, this difficulty is known as the "problem of time"; c.f. ref. [2] and [3], for recent reviews.

In this article we propose an alternative to the conventional procedures for quantizing parametrized theories. Our proposal begins with the rather trivial observation that, at the classical level, there is no observable difference between an action $S$ and the same action multiplied by a constant. Since, e.g., the action for a relativistic particle, the Nambu string, and the Einstein-Hilbert action are, respectively

$$
\begin{aligned}
S_{p} & =-m \int d \tau \sqrt{-g_{\mu \nu} \frac{d x^{\mu}}{d \tau} \frac{d x^{\nu}}{d \tau}} \\
S_{N} & =T \int d^{2} \sigma \sqrt{-\operatorname{det}\left[\eta_{\mu \nu} \frac{\partial x^{\mu}}{\partial \sigma^{i}} \frac{\partial x^{\nu}}{\partial \sigma^{j}}\right]} \\
S_{E H} & =\frac{1}{\kappa^{2}} \int d^{4} x \sqrt{g}(-R)
\end{aligned}
$$


it follows that the mass $m$, the string tension $T$, and Newton's constant $G_{N}=\kappa^{2} / 16 \pi$, which appear in the Hamiltonian constraints of the relativistic particle, string, and pure gravity theories respectively, do not appear in the Euler-Lagrange equations, which are the geodesic equation

$$
\frac{d^{2} x^{\mu}}{d s^{2}}+\Gamma_{\alpha \beta}^{\mu} \frac{d x^{\alpha}}{d s} \frac{d x^{\beta}}{d s}=0
$$

for the relativistic particle, the equation of motion

$$
\eta^{i j} \frac{\partial^{2}}{\partial \sigma^{i} \partial \sigma^{j}} x^{\mu}\left(\sigma^{1}, \sigma^{2}\right)=0
$$

for the Nambu string (in orthonormal coordinates), and Einstein's equations

$$
R_{\mu \nu}=0
$$

for pure gravity. The mass of a particle (or the tension of a string) can never be determined from its trajectory in free fall, neither can Newton's constant be determined from vacuum solutions to Einstein's equations. In the theories described by (4) these are classically indeterminate parameters, which only become relevant by introducing non-gravitational fields and forces (i.e. changing the theories).

In section 2 , below, we generalize this observation to any time-parametrized theory with hyperbolic constraints, in particular, to gravity coupled to other fields. It will be shown that there is always a classically undetermined parameter $\mathcal{E}$, which is equal to the ratio (denoted $\rightleftarrows$ ) of the kinetic and potential terms in the constrained Hamiltonian

$$
-\mathcal{E}=\mathbb{E}[p, q]=\frac{\operatorname{Kinetic}[p, q]}{\text { Potential }[q]}
$$

We will also show that replacing the usual (constrained) Hamiltonian $H[p, q]$ in Hamilton's equations by the expression $\mathbb{E}[p, q]$ generates, in configuration space (superspace), the usual classical solutions of the theory.

Our proposal for resolving the time problem in quantum gravity is based on this reasoning: since $\mathcal{E}$ is indeterminate at the classical level, we see no reason that it must be regarded as a fixed (bare) parameter at the quantum level. Rather, let $-\mathcal{E}$ denote the possible eigenvalues of the operator $Æ$. Because $Æ \neq 0$ and, as shown in section 2 , the Poisson brackets

$$
\partial_{\tau} Q=\{Q, \notin\}
$$

generate dynamics at the classical level, it is proposed that $Æ$ is also the appropriate evolution operator to use at the quantum level, i.e.

$$
\partial_{\tau}<Q>=-<\frac{i}{\hbar}[Q, \circledast]>
$$


The Hilbert space of physical states is spanned by stationary states $\Phi_{\mathcal{E}}$

$$
Æ \Phi_{\mathcal{E}}=-\mathcal{E} \Phi_{\mathcal{E}}
$$

and this time-independent Schrödinger equation will be recognized as just the usual Hamiltonian constraint with a given value of the parameter $\mathcal{E}$, which varies among stationary states.

In section 3 we rederive the $Æ$ evolution operator in a completely different way, based on a transfer-matrix quantization of parametrized theories. This section is a review and extension of the approach suggested recently by one of us in ref. [4], which leads to the same picture as that advocated in section 2. The transfer matrix approach has the advantage of fixing the operator-ordering in $\mathbb{E}[p, q]$ and the functional integration measure, for parametrized theories with a discrete number of degrees of freedom.

Section 4 is devoted to a discussion of the semiclassical correspondence between (90) and (10), and in particular the relation between the evolution parameter $\tau$ that appears in those equations, and the "many-fingered time" of general relativity. We argue that the space of physical states includes states which are sharply peaked (at a particular value of $\tau$ ) around a given 3-geometry. In such states, the dispersion around the given 3 -geometry is, crudely speaking, inversely proportional to the dispersion in $\mathcal{E}$.

We also argue that a dispersion in $\mathcal{E}$, which is characteristic of non-stationary states, would appear experimentally as an inherent uncertainty in the phenemonological value of Planck's constant. Unfortunately, we are unable to place on this uncertainty a reliable lower bound.

In section 5 we illustrate our formalism by solving a simple minisuperspace model, and display the "wavepacket of the Universe" evolving through the stages of expansion

of the scale factor, recollapse, and reexpansion. Section 6 contains some concluding remarks.

\section{The Kinetic/Potential Ratio}

We consider first a time-parametrized theory with $D$ degrees of freedom, described by the action

$$
\begin{aligned}
S & =\int d t\left(p_{a} \frac{d q^{a}}{d t}-N \mathcal{H}\right) \\
\mathcal{H} & =\frac{1}{2 m_{0}} G^{a b} p_{a} p_{b}+m_{0} V(q)
\end{aligned}
$$


where the "supermetric" $G_{a b}$ has Lorentzian signature $\{-++\ldots+\}$. For $V(q)=$ const., this action characterizes the motion of a relativistic particle in the spacetime metric $G_{a b}$; other choices of $V$ arise in minisuperspace models of quantum gravity. Making the rescaling $p_{a} \rightarrow p_{a} / \sqrt{\mathcal{E}}$ (an extended canonical transformation) the action becomes

$$
\begin{aligned}
S & =\frac{1}{\sqrt{\mathcal{E}}} \int d t\left[p_{a} \frac{d q^{a}}{d t}-N\left(\frac{1}{2 \sqrt{\mathcal{E}} m_{0}} G^{a b} p_{a} p_{b}+\sqrt{\mathcal{E}} m_{0} V\right)\right] \\
& =\frac{1}{\sqrt{\mathcal{E}}} S\left[m=\sqrt{\mathcal{E}} m_{0}\right]
\end{aligned}
$$

It is clear from this equation that the trajectory $q^{a}(t)$ in configuration space connecting two arbitrary points $q_{0}^{a}$ and $q_{1}^{a}$ cannot depend on the value of $m_{0}$, which is therefore classically indeterminate. We may also write

$$
S=\frac{1}{\sqrt{\mathcal{E}}} S^{\mathcal{E}}
$$

where

$$
\begin{aligned}
S^{\mathcal{E}} & =\int d t\left(p_{a} \frac{d q^{a}}{d t}-N \mathcal{H}^{\mathcal{E}}\right) \\
\mathcal{H}^{\mathcal{E}} & =\frac{1}{2 \sqrt{\mathcal{E}} m_{0}} G^{a b} p_{a} p_{b}+\sqrt{\mathcal{E}} m_{0} V
\end{aligned}
$$

Suppose $S$ is stationary for a trajectory in phase space $q^{a}(t)=Q^{a}(t), \quad p_{a}(t)=$ $P_{a}(t), \quad N(t)=\mathcal{N}(t)$, which connects the generalized coordinates $q_{0}^{a}$ and $q_{1}^{a}$. It follows that $S^{\mathcal{E}}$ is stationary for $q^{a}(t)=Q^{a}(t), \quad p_{a}(t)=\sqrt{\mathcal{E}} P_{a}(t), \quad N(t)=\mathcal{N}(t)$. This means that the trajectory in configuration space $q^{a}(t)=Q^{a}(t)$ connecting $q_{0}^{a}$ and $q_{1}^{a}$ will be the same whether the fundamental action is taken to be $S$ or $S^{\mathcal{E}}$. It is in this sense that the parameter $\mathcal{E}$ is indeterminate, since trajectories in configuration space generated by

$$
\mathcal{H}^{\mathcal{E}}=0 \quad \frac{\partial q^{a}}{\partial t}=N \frac{\partial \mathcal{H}^{\mathcal{E}}}{\partial p_{a}} \quad \frac{\partial p_{a}}{\partial t}=-N \frac{\partial \mathcal{H}^{\mathcal{E}}}{\partial q^{a}}
$$

will be independent of $\mathcal{E}$.

Let us rewrite the $\mathcal{H}^{\mathcal{E}}=0$ constraint as

$$
-\mathcal{E}=Æ[p, q] \equiv \frac{\frac{1}{2 m_{0}} G^{a b} p_{a} p_{b}}{m_{0} V}
$$

It is easy to show that the configuration space trajectories generated by treating $\mathbb{E}[p, q]$ as though it were a Hamiltonian

$$
Æ=-\mathcal{E} \quad \frac{\partial q^{a}}{\partial \tau}=\frac{\partial Æ}{\partial p_{a}} \quad \frac{\partial p_{a}}{\partial \tau}=-\frac{\partial Æ}{\partial q^{a}}
$$


are equivalent to the solutions generated by (16). [ The first of these equations, $\mathbb{E}=$ $-\mathcal{E}$, is just the Hamiltonian constraint $\mathcal{H}^{\mathcal{E}}=0$. The second equation is

$$
\begin{aligned}
\frac{\partial q^{a}}{\partial \tau} & =\frac{\partial Æ}{\partial p_{a}} \\
& =\frac{\sqrt{\mathcal{E}}}{m_{0} V} \frac{\partial}{\partial p_{a}} \mathcal{H}^{\mathcal{E}}
\end{aligned}
$$

and for the third equation we have

$$
\begin{aligned}
\frac{\partial p_{a}}{\partial \tau} & =-\frac{\partial \Subset}{\partial q^{a}} \\
& =-\frac{1}{m_{0} V}\left[\frac{\partial}{\partial q^{a}}\left(\frac{1}{2 m_{0}} G^{a b} p_{a} p_{b}\right)-\frac{\frac{1}{2 m_{0}} G^{a b} p_{a} p_{b}}{m_{0} V} \frac{\partial}{\partial q^{a}}\left(m_{0} V\right)\right] \\
& =-\frac{1}{m_{0} V} \frac{\partial}{\partial q^{a}}\left[\frac{1}{2 m_{0}} G^{a b} p_{a} p_{b}+\mathcal{E} m_{0} V\right] \\
& =-\frac{\sqrt{\mathcal{E}}}{m_{0} V} \frac{\partial}{\partial q^{a}} \mathcal{H}^{\mathcal{E}}
\end{aligned}
$$

Suppose $\bar{q}(\tau), \bar{p}(\tau)$ is some particular solution of (19), (20), and $Æ=-\mathcal{E}$. Then, choosing

$$
N(\tau)=\frac{1}{V[\bar{q}(\tau)]}
$$

and rescaling $\tau$ by $\sqrt{\mathcal{E}} / m_{0}$ to give $\tau$ the conventional units of time, we see that this is also a solution of the Hamilton's equations and constraint (16). It follows that, in general, the Poisson bracket evolution equation

$$
\partial_{\tau} O=\{O, \circledast \mathbb{E}\}
$$

supplemented by $Æ=-\mathcal{E}$ will generate time evolution which is equivalent, up to a time reparametrization, to evolution generated by

$$
\partial_{t} O=\left\{O, N \mathcal{H}^{\mathcal{E}}\right\}
$$

supplemented by $\mathcal{H}^{\mathcal{E}}=0$.

The extension of these remarks to general relativity is fairly straightforward. Let us denote the action of a generally covariant field theory as

$$
\begin{aligned}
S & =\int d^{4} x\left[p_{a} \frac{\partial q^{a}}{\partial t}-N \mathcal{H}_{x}-N_{i} \mathcal{H}_{x}^{i}\right] \\
\mathcal{H}_{x} & =\kappa^{2} G^{a b} p_{a} p_{b}+\sqrt{g} U(q)
\end{aligned}
$$

\footnotetext{
${ }^{3}$ It should be noted that, since $Æ$ is dimensionless, the evolution parameter $\tau$ in eq. (18) has units of action.
} 
where the $q^{a}(x)$ are the set of all fields, gravitational and non-gravitational, $G_{a b}$ is the supermetric, $N$ and $N_{i}$ the lapse and shift functions, and $\mathcal{H}_{x}^{i}$ are the supermomenta, linear in the canonical momenta $p_{a}(x)$. It is convenient to rescale all non-gravitational fields by an appropriate power of $\kappa$ so that all $q^{a}(x)$, and all $G^{a b}$, are dimensionless. In the special case of pure gravity, we would identify

$$
\begin{aligned}
& \{a=1-6\} \leftrightarrow\{(i, j), i \leq j\} \\
& q^{a}(x) \leftrightarrow g_{i j}(x) \\
& p_{a}(x) \leftrightarrow\left\{\begin{aligned}
p^{i j}(x) & (i=j) \\
2 p^{i j}(x) & (i<j)
\end{aligned}\right. \\
& G_{a b}(x) \leftrightarrow G^{i j n m}(x) \\
& U \leftrightarrow \frac{1}{\kappa^{2}}\left(-{ }^{3} R\right)
\end{aligned}
$$

By the same rescaling $p_{a} \rightarrow p_{a} / \sqrt{\mathcal{E}}$ as before, the action $S$ is equivalent at the classical level to the action

$$
\begin{aligned}
S^{\mathcal{E}} & =\int d^{4} x\left[p_{a} \frac{\partial q^{a}}{\partial t}-N \mathcal{H}_{x}^{\mathcal{E}}-N_{i} \mathcal{H}_{x}^{i}\right] \\
\mathcal{H}_{x}^{\mathcal{E}} & =\frac{\kappa^{2}}{\sqrt{\mathcal{E}}} G^{a b} p_{a} p_{b}+\sqrt{\mathcal{E}} \sqrt{g} U(q)
\end{aligned}
$$

Variation of $S^{\mathcal{E}}$ with respect to the lapse gives the Hamiltonian constraint

$$
\mathcal{H}_{x}^{\mathcal{E}}=\frac{\kappa^{2}}{\sqrt{\mathcal{E}}} G^{a b} p_{a} p_{b}+\sqrt{\mathcal{E}} \sqrt{g} U(q)=0
$$

which, multiplying again by an arbitrary function $N(x)$, we write in the form

$$
-\mathcal{E}=\frac{\int d^{3} x N \kappa^{2} G^{a b} p_{a} p_{b}}{\int d^{3} x N \sqrt{g} U(q)}
$$

valid for any $N(x)$. Because of the supermomenta constraints $\mathcal{H}_{x}^{i}=0$, we can add a term proportional to the supermomenta to the ratio in (28) without changing its value. Define

$$
\begin{aligned}
\rightleftarrows\left[p, q, N, N_{i}\right] & \equiv \int d^{3} x\left[\frac{N \kappa^{2} G^{a b} p_{a} p_{b}}{\int d^{3} x^{\prime} \sqrt{g} N U(q)}+\frac{1}{m_{P}} N_{i} \mathcal{H}_{x}^{i}\right] \\
& =\frac{1}{m_{P}} \int d^{3} x\left\{\tilde{N} \kappa^{2} G^{a b} p_{a} p_{b}+N_{i} \mathcal{H}_{x}^{i}\right\}
\end{aligned}
$$

where

$$
\tilde{N}(x) \equiv \frac{m_{P} N(x)}{\int d^{3} x^{\prime} \sqrt{g} N U(q)}
$$


and $m_{P}$ is an arbitrary mass parameter. We will now show that $Æ$ can itself be reinterpreted as a Hamiltonian, in the sense that the classical orbits in configuration space obtained from the corresponding Hamilton's equations are identical to the extrema of the action (24).

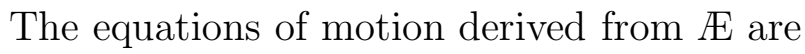

$$
\begin{aligned}
& \frac{d q^{a}(x)}{d \tau}=\frac{\delta \circledast}{\delta p_{a}(x)} \\
& =\frac{1}{m_{P}} \int d^{3} x^{\prime}\left[\tilde{N}\left(x^{\prime}\right) \frac{\delta}{\delta p_{a}(x)}\left(\kappa^{2} G^{c d} p_{c} p_{d}\right)_{x^{\prime}}+N_{i}\left(x^{\prime}\right) \frac{\delta}{\delta p_{a}(x)} \mathcal{H}_{x^{\prime}}^{i}\right] \\
& \frac{d p_{a}(x)}{d \tau}=-\frac{\delta Æ}{\delta q^{a}(x)} \\
& =-\frac{1}{m_{P}}\left\{\int d^{3} x^{\prime}\left[\tilde{N}\left(x^{\prime}\right) \frac{\delta}{\delta q^{a}(x)}\left(\kappa^{2} G^{c d} p_{c} p_{d}\right)_{x^{\prime}}+N_{i}\left(x^{\prime}\right) \frac{\delta}{\delta q^{a}(x)} \mathcal{H}_{x^{\prime}}^{i}\right]\right. \\
& \left.-\frac{\int N \kappa^{2} G^{c d} p_{c} p_{d}}{\left(\int N \sqrt{g} U\right)^{2}} \int d^{3} x^{\prime} m_{P} N\left(x^{\prime}\right) \frac{\delta}{\delta q^{a}(x)}(\sqrt{g} U)_{x^{\prime}}\right\} \\
& =-\frac{1}{m_{P}} \int d^{3} x^{\prime}\left[\tilde{N}\left(x^{\prime}\right) \frac{\delta}{\delta q^{a}(x)}\left(\kappa^{2} G^{c d} p_{c} p_{d}\right)_{x^{\prime}}-\tilde{N}\left(x^{\prime}\right) Æ \frac{\delta}{\delta q^{a}(x)}(\sqrt{g} U)_{x^{\prime}}\right. \\
& \left.+N_{i}\left(x^{\prime}\right) \frac{\delta}{\delta q^{a}(x)} \mathcal{H}_{x^{\prime}}^{i}\right] \\
& 0=\frac{\delta Æ}{\delta N(x)} \\
& =\frac{1}{\int N \sqrt{g} U}\left(\kappa^{2} G^{a b} p_{a} p_{b}\right)_{x}-\frac{\left(\int N \kappa^{2} G^{a b} p_{a} p_{b}\right)}{\left(\int N \sqrt{g} U\right)^{2}}(\sqrt{g} U)_{x} \\
& 0=\frac{\delta \circledast ~}{\delta N_{i}(x)}=\frac{1}{m_{P}} \mathcal{H}_{x}^{i}
\end{aligned}
$$

The value of $Æ$ is of course a constant of this motion, denoted $Æ=-\mathcal{E}$, and $\tau$ is dimensionless. From the definition of $\mathcal{H}_{x}^{\mathcal{E}}$ in eq. (26), and rescaling $t=\sqrt{\mathcal{E}} \tau / m_{P}$ (to give $t$ the dimensions of length), the equations of motion (31) become

$$
\begin{aligned}
\frac{d q^{a}(x)}{d t} & =\int d^{3} x^{\prime}\left[\tilde{N}\left(x^{\prime}\right) \frac{\delta}{\delta p_{a}(x)} \mathcal{H}_{x^{\prime}}^{\mathcal{E}}+\tilde{N}_{i}\left(x^{\prime}\right) \frac{\delta}{\delta p_{a}(x)} \mathcal{H}_{x^{\prime}}^{i}\right] \\
\frac{d p_{a}(x)}{d t} & =-\int d^{3} x^{\prime}\left[\tilde{N}\left(x^{\prime}\right) \frac{\delta}{\delta q^{a}(x)} \mathcal{H}_{x^{\prime}}^{\mathcal{E}}+\tilde{N}_{i}\left(x^{\prime}\right) \frac{\delta}{\delta q^{a}(x)} \mathcal{H}_{x^{\prime}}^{i}\right] \\
\mathcal{H}_{x}^{\mathcal{E}} & =\frac{\kappa^{2}}{\sqrt{\mathcal{E}}} G^{a b} p_{a} p_{b}+\sqrt{\mathcal{E}} \sqrt{g} U=0 \\
\mathcal{H}_{x}^{i} & =0
\end{aligned}
$$

where the shift function is $\tilde{N}_{i} \equiv N_{i} / \sqrt{\mathcal{E}}$. Comparison of the equations of motion (32) to the Hamiltonian equations of motion that would be derived from $S^{\mathcal{E}}$ of eq. (26) shows 
that they are the same equations, apart from the restriction of the lapse function $\tilde{N}$ in (32) to satisfy

$$
\int d^{3} x \sqrt{g} \tilde{N} U=m_{P}
$$

The trajectory in configuration space is independent of the choice of lapse; it is also independent, as noted above, of the choice of $\mathcal{E}$.

We can therefore conclude that $\mathbb{E}\left[p, q, N, N_{i}\right]$, viewed as a Hamiltonian, generates the same classical dynamics as the more conventional Hamiltonian form

$$
H=\int d^{3} x\left[N \mathcal{H}_{x}+N_{i} \mathcal{H}_{x}^{i}\right]
$$

The crucial difference between $\mathbb{E}$ and $H$ is that $H$ is constrained to vanish, in the standard formulation, whereas the value of $\mathbb{E}$ is unconstrained: the constant $\mathcal{E}$ can take on any value on an orbit, depending only on the initial choice of $\{p, q\}$. Obviously, this difference is quite important at the quantum level. Instead of the usual Wheeler-de Witt constraint equation

$$
H \Psi[q]=0
$$

we will obtain a Schrödinger equation

$$
Æ \Psi[q, \tau]=i \hbar \partial_{\tau} \Psi[q, \tau]
$$

Several questions arise immediately:

1. If (36) is the dynamical equation of quantum gravity, what has become of the Poincaré algebra of constraints which represents the diffeomorphism invariance of the theory?

2. What is the relationship of the Schrödinger equation (36), based on the Æ-operator, to path-integral quantization?

3. What is the operator ordering in $\mathbb{E}\left[p, q, N, N_{i}\right]$, and what integration measure should be used for the inner product of states?

4. The time-evolution parameter $\tau$ in the Schrödinger equation is only a single variable; how is it related to the "many-fingered time" of general relativity?

To answer the first question (the others will be dealt with in subsequent sections), let us note that physical states $\Psi[q, \tau]$ in the Schrödinger representation must be independent of the functions $N$ and $N_{i}$. Expanding an arbitrary $\Psi$ in stationary states

$$
\Psi[q, \tau]=\sum_{\mathcal{E}} a_{\mathcal{E}} \Phi_{\mathcal{E}}[q] e^{i \mathcal{E} \tau / \hbar}
$$


where

$$
Æ \Phi_{\mathcal{E}}[q]=-\mathcal{E} \Phi_{\mathcal{E}}[q]
$$

the condition of $N_{i}$-independence gives

$$
\begin{aligned}
0 & =m_{P} \frac{\delta}{\delta N_{i}(x)} \circledast \Phi_{\mathcal{E}} \\
& =\mathcal{H}_{x}^{i} \Phi_{\mathcal{E}}
\end{aligned}
$$

while $N$-independence requires

$$
\begin{aligned}
0 & =\frac{\delta}{\delta N(x)} \Subset \Phi_{\mathcal{E}} \\
& =\frac{1}{\int d^{3} x^{\prime} \sqrt{g} N U}\left[\kappa^{2} G^{a b} p_{a} p_{b}-\sqrt{g} U Æ\right]_{x} \Phi_{\mathcal{E}} \\
& =\frac{\sqrt{\mathcal{E}}}{\int d^{3} x^{\prime} \sqrt{g} N U} \mathcal{H}_{x}^{\mathcal{E}} \Phi_{\mathcal{E}}
\end{aligned}
$$

where we have provisionally taken an operator-ordering in $Æ$ with momenta to the right, and also used the supermomentum constraint (39). Therefore, the Hilbert space of physical states consists of linear combinations of Æ-eigenstates

$$
\Psi_{\text {phys }}[q]=\sum_{\mathcal{E}} a_{\mathcal{E}} \Phi_{\mathcal{E}}[q]
$$

each of which satisfies the constraints

$$
\begin{aligned}
& \mathcal{H}_{x}^{\mathcal{E}} \Phi_{\mathcal{E}}=\frac{1}{\sqrt{\mathcal{E}}}\left[\kappa^{2} G^{a b} p_{a} p_{b}+\mathcal{E} \sqrt{g} U\right]_{x} \Phi_{\mathcal{E}}=0 \\
& \mathcal{H}_{x}^{i} \Phi_{\mathcal{E}}=0
\end{aligned}
$$

Apart from the parameter $\mathcal{E}$, these are the standard constraints on physical states of quantum gravity. As is customary in this subject, we assume that there exists some operator-ordering such that the commutators of operators $\mathcal{H}_{x}^{\mathcal{E}}, \mathcal{H}_{x}^{i}$ close on the Poincaré algebra, as do the Poisson brackets of the corresponding classical quantities. Given this assumption, the physical states of the form (41) satisfy, in our formulation, the generalized Dirac constraints

$$
\begin{aligned}
\left\{\left[\kappa^{2} G^{a b} p_{a} p_{b}\right]_{x}-[\sqrt{g} U]_{x} \mathbb{E}\right\} \Psi_{\text {phys }} & =0 \\
\mathcal{H}_{x}^{i} \Psi_{\text {phys }} & =0
\end{aligned}
$$

In the case of pure quantum gravity, the $\mathcal{E}$ parameter has a simple interpretation. The Hamiltonian constraint becomes

$$
\left\{\frac{\kappa^{2}}{\sqrt{\mathcal{E}}} G_{i j k l} p^{i j} p^{k l}+\frac{\sqrt{\mathcal{E}}}{\kappa^{2}} \sqrt{g}\left(-{ }^{3} R\right)\right\} \Phi_{\mathcal{E}}=0
$$


and therefore

$$
G_{N}=\frac{\kappa^{2}}{16 \pi \sqrt{\mathcal{E}}}
$$

is the effective Newton's constant for the degenerate subspace of physical states satisfying $Æ \Phi=-\mathcal{E} \Phi$. The space of physical states is spanned by states $\Phi_{\mathcal{E}}$ which satisfy the standard Hamiltonian and supermomentum constraints, but with a different value of Newton's constant associated with each $\mathcal{E}$. In general, physical states are not eigenstates of Newton's constant.

To summarize our proposal for pure gravity: since Newton's constant is indeterminate at the classical level, we see no reason that it is necessarily a fixed bare parameter at the quantum level. Instead, treating $G_{N}$ as a quantum number allows for a vast extension of the space of physical states. It is this extension which we propose to exploit, as seen in eq. (36), to resolve the time problem in quantum gravity. In the general case of gravity coupled to other fields, it is not Newton's constant per se but rather the kinetic/potential ratio in the constrained Hamiltonian that is classically indeterminate. Regarding this ratio as a q-number leads again to an extension of the space of physical states and, as we will argue further below, to a solution of the problem of time.

\section{The Transfer-Matrix Formulation}

We now give an alternate derivation of the Schrödinger equation (36) for parametrized systems, following the transfer-matrix formulation proposed by one of us in ref. 迎. The advantage of this approach, apart from making the connection to path-integral formalism, is that it also fixes the measure and operator-ordering, at least for systems with a discrete number of degrees of freedom.

The transfer matrix $\mathcal{T}_{\epsilon}$ for non-parametrized systems, in statistical mechanics and Euclidean quantum mechanics, is an operator which evolves states by a time-step $\Delta t=\epsilon$

$$
\begin{aligned}
\psi\left(q^{\prime}, t+\epsilon\right) & =\mathcal{T}_{\epsilon} \psi\left(q^{\prime}, t\right) \\
& =\int d^{D} q \mu(q) \exp \left[-S_{\epsilon}\left(q^{\prime}, q\right)\right] \psi(q, t)
\end{aligned}
$$

Denoting by $S\left[q_{2}, q_{1} ; \Delta t\right]$ the action of a classical solution $q(t)$ running between the initial point $q_{1}$ at time $t$ and final $q_{2}$ at time $t+\Delta t$, the expression $S_{\epsilon}$ in Euclidean quantum mechanics is given by the continuation of $S$ to imaginary time lapse

$$
S_{\epsilon}\left(q_{2}, q_{1}\right) \equiv i S\left[q_{2}, q_{1} ; i \epsilon\right] / \hbar
$$

The measure $\mu(q)$ is defined such that $\mathcal{T}_{\epsilon}$ is an identity operator as $\epsilon \rightarrow 0$. The usual 
quantum-mechanical Hamiltonian is essentially the logarithm of the transfer matrix

$$
H=\lim _{\epsilon \rightarrow 0}\left(-\frac{\hbar}{\epsilon}\right) \ln \left[\mathcal{T}_{\epsilon}\right]
$$

and the continuum (Euclidean) path integral is defined as the limit of a product of transfer matrices

$$
\begin{aligned}
\psi\left(q^{\prime}, t_{1}\right) & =\int D q\left(t_{0} \leq t<t_{1}\right) e^{-S[q(t)] / \hbar} \psi\left(q, t_{0}\right) \\
& =\lim _{\epsilon \rightarrow 0} \int \prod_{n=0}^{N-1} d^{D} q_{n} \mu\left(q_{n}\right) \exp \left[-\sum_{n=0}^{N-1} S_{\epsilon}\left(q_{n+1}, q_{n}\right)\right] \psi\left(q_{0}, t_{0}\right) \\
& =\lim _{\epsilon \rightarrow 0}\left(\mathcal{T}_{\epsilon}\right)^{N} \psi\left(q^{\prime}, t_{0}\right) \quad \text { where } \quad N \equiv \frac{t_{1}-t_{0}}{\epsilon}
\end{aligned}
$$

Now let us again consider parametrized theories of the form

$$
\begin{aligned}
S & =\int d t\left(p_{a} \frac{d q^{a}}{d t}-N \mathcal{H}\right) \\
\mathcal{H} & =\frac{1}{2 m} G^{a b} p_{a} p_{b}+m V(q)
\end{aligned}
$$

For parametrized theories of this sort, the transfer matrix formalism above breaks down at eq. (47). The problem is that the action $S\left[q_{2}, q_{1}, G_{a b}\right]$ of a classical trajectory connecting initial coordinates $q_{1}$ and final coordinates $q_{2}$ depends only on those coordinates (and choice of supermetric $G_{a b}$ ); there is no additional dependence on a time lapse $\Delta \tau$, and the values of parameters $\tau_{1}$ and $\tau_{2}$ that happen to be associated with the initial and final coordinates are irrelevant. A transfer matrix $\mathcal{T}_{\epsilon}$ based on (47) would then be independent of $\epsilon$; this is one of the obstacles encountered in trying to apply directly the path-integral approach to find the time-evolution of physical states in parametrized theories.

It is premature to conclude, however, from the failure of (47), that the transfer matrix concept is meaningless for parametrized theories. Let us denote an orbit in Hilbert space by $\psi(q, \tau)$, and require that the transfer matrix $\mathcal{T}_{\epsilon}$ evolves states in the parameter $\tau$ such that the orbit of the center of the wave packet

$$
<q^{a}(\tau)>\equiv<\psi(q, \tau)\left|q^{a}\right| \psi(q, \tau)>
$$

obeys an appropriate Ehrenfest principle. We will show, following ref. [4], that this is achieved by replacing (47) in the definition of the transfer matrix by

$$
S_{\epsilon}\left(q_{2}, q_{1}\right)=i S\left[q_{2}, q_{1}, G_{a b}^{E}\right] / \sqrt{\epsilon \hbar}
$$

where the Euclidean rotation $\Delta t \rightarrow i \Delta t$ in (47) is replaced by a rotation of the signature of the supermetric

$$
\begin{aligned}
G_{a b}(q) & =E_{a}^{i}(q) \eta_{i j} E_{b}^{j}(q) \\
G_{a b}^{E} & \equiv \operatorname{sign}[V(q)] E_{a}^{i}(q) \delta_{i j} E_{b}^{j}(q)
\end{aligned}
$$


The reason for this peculiar rotation of signature is to ensure that $S_{\epsilon}\left(q_{2}, q_{1}\right)$ is real for all choices of $q_{1}$ and $q_{2}$. To show this, we first compute $S\left[q, q+\Delta q, G_{a b}\right]$, beginning from Hamilton's equation

$$
\dot{q}^{a}=N \frac{\partial \mathcal{H}}{\partial p_{a}}=\frac{N}{m} G^{a b} p_{b} \Rightarrow p_{a}=\frac{m}{N} G_{a b} \dot{q}^{b}
$$

Insert this into the constraint equation $\mathcal{H}=0$

$$
\frac{1}{2 N^{2}} G_{a b} \dot{q}^{a} \dot{q}^{b}+V=0
$$

and solve for the lapse

$$
N=\left[-\frac{1}{2 V} G_{a b} \dot{q}^{a} \dot{q}^{b}\right]^{1 / 2}
$$

Then

$$
\begin{aligned}
S\left[q, q+\Delta q, G_{a b}\right] & =\int_{0}^{\Delta t} d t \frac{m}{N} G_{a b} \dot{q}^{a} \dot{q}^{b} \\
& =-m \int_{0}^{\Delta t} d t \sqrt{-2 V G_{a b} \dot{q}^{a} \dot{q}^{b}} \\
& =-m \sqrt{-2 V G_{a b} \Delta q^{a} \Delta q^{b}} \\
& =-m \sqrt{-\mathcal{G}_{a b} \Delta q^{a} \Delta q^{b}}
\end{aligned}
$$

where we define a modified supermetric

$$
\mathcal{G}_{a b} \equiv 2 V G_{a b}
$$

It is clear that if the signature of $G_{a b}$ is Lorentzian, then $S\left[q, q+\Delta q, G_{a b}\right]$ is not necessarily real. However, upon the signature rotation (53), the function

$$
\begin{aligned}
S_{\epsilon}(q, q+\Delta q) & =m \sqrt{2 V G_{a b}^{E} \Delta q^{a} \Delta q^{b}} / \sqrt{\epsilon \hbar} \\
& =m \sqrt{\mathcal{G}_{a b}^{E} \Delta q^{a} \Delta q^{b}} / \sqrt{\epsilon \hbar}
\end{aligned}
$$

is strictly real-valued for any choice of $\Delta q^{a}$.

The integration measure $\mu$ is chosen to be

$$
\mu^{-1}\left(q^{\prime}\right)=(\sqrt{\epsilon \hbar})^{D} \lim _{\epsilon \rightarrow 0} \int \frac{d^{D} q}{(\sqrt{\epsilon \hbar})^{D}} \exp \left(-S_{\epsilon}\left(q^{\prime}, q\right)\right)
$$

This choice ensures that $\mathcal{T}_{\epsilon} \rightarrow 1$ in the $\epsilon \rightarrow 0$ limit, and that the symmetries of the action are reflected in the measure.

The equations (52),(53), (60), when inserted into eq. (46), define a transfer matrix $\mathcal{T}_{\epsilon}$ for the parametrized, signature-rotated theory, which in general depends on $\mathcal{G}^{E}$ and 
$\operatorname{det}\left[\mathcal{G}^{E}\right]$. The final step is to undo the signature rotation in computing the corresponding evolution operator $\rightleftarrows$

$$
\rightleftarrows=\left[\lim _{\epsilon \rightarrow 0}\left(-\frac{\hbar}{\epsilon}\right) \ln \left[\mathcal{T}_{\epsilon}\right]\right]\left\{\begin{array}{c}
\mathcal{G}^{E} \rightarrow \mathcal{G} \\
\operatorname{det}\left(\mathcal{G}^{E}\right) \rightarrow|\operatorname{det}(\mathcal{G})|
\end{array}\right\}
$$

and the evolution of states is given by

$$
i \hbar \partial_{\tau} \psi(q, \tau)=Æ \psi(q, \tau)
$$

We can now evaluate $\mathcal{T}_{\epsilon}$ and $Æ$ for actions of the form $(50)$, leading to the function $S_{\epsilon}$ shown in (59). Begin by introducing Riemann normal coordinates $\xi^{a}$ around $q^{\prime a}=$ $q^{a}-\Delta q^{a}$, which bring $\mathcal{G}_{a b}^{E}=\delta_{a b}$ at $\xi^{a}=0$. In these coordinates

$$
S_{\epsilon}(0, \xi)=m \sqrt{\delta_{a b} \xi^{a} \xi^{b}+O\left(\xi^{5}\right)} / \sqrt{\epsilon \hbar}
$$

The $O\left(\xi^{5}\right)$ terms will not contribute to $Æ$ in the $\epsilon \rightarrow 0$ limit, and can be dropped. The measure is then

$$
\begin{aligned}
\mu^{-1}\left(q^{\prime}\right) & =(\sqrt{\epsilon \hbar})^{D} \lim _{\epsilon \rightarrow 0} \int \frac{d^{D} \xi}{(\sqrt{\epsilon \hbar})^{D}} \operatorname{det}\left[\frac{\partial \Delta q^{\mu}}{\partial \xi^{\nu}}\right] \exp [-m|\xi| / \sqrt{\epsilon \hbar}] \\
& =\frac{2 \pi^{D / 2}}{\Gamma(D / 2)}(D-1) !\left(\frac{\sqrt{\epsilon \hbar}}{m}\right)^{D} \frac{1}{\sqrt{\mathcal{G}^{E}\left(q^{\prime}\right)}}=\frac{\sigma}{\sqrt{\mathcal{G}^{E}\left(q^{\prime}\right)}}
\end{aligned}
$$

The operation of the transfer matrix (46) becomes

$$
\begin{aligned}
\psi\left(q^{\prime}, \tau+\epsilon\right)= & \int \frac{d^{D} \Delta q}{\sigma} \sqrt{\mathcal{G}^{E}\left(q^{\prime}+\Delta q\right)} \exp \left[-m \sqrt{\mathcal{G}_{\alpha \beta}^{E} \Delta q^{\alpha} \Delta q^{\beta}} / \sqrt{\epsilon \hbar}\right] \psi\left(q^{\prime}+\Delta q, \tau\right) \\
= & \int \frac{d^{D} \xi}{\sigma}\left(1-\frac{1}{6} \mathcal{R}_{\alpha \beta} \xi^{\alpha} \xi^{\beta}+\ldots\right) \exp \left[-\frac{m|\xi|}{\sqrt{\epsilon \hbar}}\right]\left\{\psi\left(q^{\prime}, \tau\right)+\frac{\partial \psi}{\partial \xi^{\mu}} \xi^{\mu}\right. \\
& \left.+\frac{1}{2} \frac{\partial^{2} \psi}{\partial \xi^{\mu} \partial \xi^{\nu}} \xi^{\mu} \xi^{\nu}+O\left(\xi^{3}\right)\right\} \\
= & {\left[1+\epsilon \hbar \frac{D+1}{2 m^{2}} \partial^{\mu} \partial_{\mu}-\epsilon \hbar \frac{D+1}{6 m^{2}} \mathcal{R}+O\left(\epsilon^{2}\right)\right] \psi\left(q^{\prime}(\xi), \tau\right) }
\end{aligned}
$$

where $\mathcal{R}$ is the curvature scalar formed from the modified supermetric $\mathcal{G}_{a b}^{E}$ of eq. (58), which has been rotated to Euclidean signature. Transforming back from Riemann normal coordinates, we have

$$
\mathcal{T}_{\epsilon}=1+\epsilon\left[\frac{D+1}{2 m^{2}} \hbar \frac{1}{\sqrt{\mathcal{G}^{E}}} \frac{\partial}{\partial q^{n}} \sqrt{\mathcal{G}^{E}} \mathcal{G}^{E n m} \frac{\partial}{\partial q^{m}}-\hbar \frac{D+1}{6 m^{2}} \mathcal{R}\right]+O\left(\epsilon^{2}\right)
$$

from which we extract, according to (61)

$$
\rightleftarrows=-\frac{D+1}{2 m^{2}} \hbar^{2} \frac{1}{\sqrt{|\mathcal{G}|}} \frac{\partial}{\partial q^{n}} \sqrt{|\mathcal{G}|} \mathcal{G}^{n m} \frac{\partial}{\partial q^{m}}+\hbar^{2} \frac{D+1}{6 m^{2}} \mathcal{R}
$$


where $\mathcal{G} \equiv \operatorname{det}\left(\mathcal{G}_{m n}\right)$, and $G_{m n}$ has been rotated back to Lorentzian signature.

The classical quantity $Æ_{c l}$ corresponding to the operator $\Subset$ is obtained by replacing derivatives with c-number momenta

$$
Æ_{c l}\left[q^{a}, p_{a}\right]=\lim _{\hbar \rightarrow 0} \circledast\left[q^{a},-i \hbar \frac{\partial}{\partial q^{a}} \rightarrow p_{a}\right]
$$

which gives

$$
\rightleftarrows_{c l}=(D+1) \frac{\frac{1}{2 m} G^{a b} p_{a} p_{b}}{m V}
$$

This quantity, apart from an unimportant overall factor of $(D+1)$, is simply the Kinetic/Potential ratio introduced in the previous section. The transfer matrix approach is thus a second way of obtaining the evolution operator $\mathbb{E}$, and also provides a definite prescription for the integration measure $(\mu(q) \propto|\operatorname{det}(\mathcal{G})|)$, and operator ordering.

As a further consistency check, we note that in ordinary Euclidean quantum mechanics we may define

$$
\begin{aligned}
p \psi\left(q^{\prime}\right) & \equiv \lim _{\epsilon \rightarrow 0} \int d^{D} q \mu m \frac{\left(q^{\prime}-q\right)}{\epsilon} \exp \left[-S_{\epsilon}\left(q^{\prime}, q\right)\right] \psi(q) \\
S_{\epsilon}\left(q^{\prime}, q\right) & =\left[\frac{1}{2} m \frac{\left(q^{\prime}-q\right)^{2}}{\epsilon}+V(q) \epsilon\right] / \hbar
\end{aligned}
$$

from which it is easy to evaluate the commutator

$$
\begin{aligned}
{[q, p] \psi } & =\lim _{\epsilon \rightarrow 0} \int d^{D} q \mu\left\{q^{\prime} m \frac{\left(q^{\prime}-q\right)}{\epsilon}-m \frac{\left(q^{\prime}-q\right)}{\epsilon} q\right\} \exp \left[-S_{\epsilon}\left(q^{\prime}, q\right)\right] \psi(q) \\
& =\hbar \psi
\end{aligned}
$$

The factor $\hbar$, rather than $i \hbar$, is due to the Euclidean time. To derive the corresponding result in the parametrized theory, we begin from Hamilton's equation

$$
\begin{aligned}
\frac{\partial q^{a}}{\partial \tau} & =\frac{\partial Æ_{c l}}{\partial p_{a}} \\
& =\frac{(D+1)}{m^{2} V} G^{a b} p_{b} \quad \Rightarrow \quad p_{a}=\frac{m^{2} V}{D+1} G_{a b} \frac{\partial q^{b}}{\partial \tau}
\end{aligned}
$$

and define

$$
p_{a} \psi\left(q^{\prime}\right) \equiv \lim _{\epsilon \rightarrow 0} \int d^{D} q \mu(q) \frac{m^{2} V}{(D+1)} G_{a b}^{E} \frac{\left(q^{\prime b}-q^{b}\right)}{\epsilon} \exp \left[-S_{\epsilon}\left(q^{\prime}, q\right)\right] \psi(q)
$$

with $S_{\epsilon}$ and $\mu(q)$ given by (59) and (60). It is then easy to show that

$$
\left[q^{a}, p_{b}\right] \psi=\hbar \delta_{b}^{a} \psi
$$

as in the non-parametrized case. 
Next we consider general covariant field theories of the form (24). The main obstacle to computing $S\left[q^{\prime}, q, G_{a b}\right]$, and hence to computing the transfer matrix, is the presence of the shift functions $N_{i}$ in the classical action. Covariant derivatives of the shift functions appear in Hamilton's equation, e.g. in the case of pure gravity

$$
\frac{\partial g_{i j}}{\partial t}=2 \kappa^{2} N G_{i j n m} p^{n m}+N_{i ; j}+N_{j ; i}
$$

and these make it impossible to solve for the lapse-shift functions algebraically, in terms of $\partial_{t} g_{i j}$. To determine the lapse-shift functions, it is necessary to solve certain intractable partial differential equations. Instead, we adopt the strategy of simply setting $N_{i}=0$. In that case, of course, the supermomentum constraints are not obtained by extremizing the action, and must be recovered by imposing operator constraints of the form

$$
Q_{x}\left[q^{a}, p_{b}\right] \Psi=0
$$

on the physical states. These constraints (up to operator-ordering ambiguities) will be determined below. With the choice $N_{i}=0$, Hamilton's equations give

$$
p_{a}=\frac{1}{2 \kappa^{2} N} G_{a b} \dot{q}^{b}
$$

Inserting this into the constraint equation

$$
0=\mathcal{H}_{x}=\frac{1}{4 \kappa^{2} N^{2}} G_{a b} \dot{q}^{a} \dot{q}^{b}+\sqrt{g} U
$$

and solving for the lapse, gives

$$
N=\left[-\frac{1}{4 \kappa^{2} \sqrt{g} U} G_{a b} \dot{q}^{a} \dot{q}^{b}\right]^{1 / 2}
$$

so we have

$$
\begin{aligned}
\Delta S=S\left[q^{\prime}, q, G_{a b}\right] & =-\frac{1}{\kappa} \int d^{3} x \int_{0}^{\Delta t} d t \sqrt{-g^{\frac{1}{2}} U G_{a b} \dot{q}^{a} \dot{q}^{b}} \\
& =-\frac{1}{\kappa} \int d^{3} x\left[\sqrt{-\left(g^{\frac{1}{2}} U G_{a b}\right)_{0} \Delta q^{a} \Delta q^{b}}+O\left(\Delta q^{2}\right)\right] \\
& =-\frac{1}{\kappa} \int d^{3} x(\sqrt{g})_{0}\left[\sqrt{-\left(\mathcal{G}_{a b}\right)_{0} \Delta q^{a} \Delta q^{b}}+O\left(\Delta q^{2}\right)\right]
\end{aligned}
$$

where $\Delta q^{a}=q^{a}-q^{\prime a}$, and we define

$$
\mathcal{G}_{a b} \equiv \frac{1}{\sqrt{g}} U G_{a b}
$$

The notation $(. .)_{0}$ means that the quantity in parenthesis is to be evaluated at $\Delta q=0$; i.e. at $q^{\prime}$. 
Applying the prescriptions (46), (52), (53), the transfer matrix is formally obtained from

$$
\begin{aligned}
\psi\left(q^{\prime}, \tau+\epsilon\right)= & \int D q \mu(q) e^{-\Delta S / \sqrt{\epsilon \hbar}}\left[\psi\left(q^{\prime}\right)+\int d^{3} x\left(\frac{\delta \psi}{\delta q^{a}(x)}\right) \Delta q^{a}(x)\right. \\
& \left.+\frac{1}{2} \int d^{3} x d^{3} y\left(\frac{\delta^{2} \psi}{\delta q^{a}(x) \delta q^{b}(y)}\right) \Delta q^{a}(x) \Delta q^{b}(y)+\ldots\right] \\
= & \psi\left(q^{\prime}, \tau\right)+\epsilon\left[T_{0}+T_{1}+T_{2}\right]+O\left(\epsilon^{2}\right) \\
= & \mathcal{T}_{\epsilon} \psi\left(q^{\prime}, \tau\right)
\end{aligned}
$$

where the $T_{n}$ represent terms with $n$ functional derivatives of $\psi$. To find these terms, we need to evaluate

$$
\begin{aligned}
<\Delta q^{a}\left(x_{1}\right) \Delta q^{b}\left(x_{2}\right)>= & \int D(\Delta q)(\mu)_{0} \Delta q^{a}\left(x_{1}\right) \Delta q^{b}\left(x_{2}\right) \\
& \times \exp \left[-\frac{1}{\kappa} \int d^{3} x(\sqrt{g})_{0} \sqrt{\left(\mathcal{G}_{a b}^{E}\right)_{0} \Delta q^{a} \Delta q^{b}} / \sqrt{\epsilon \hbar}\right]
\end{aligned}
$$

Unfortunately this quantity is highly singular, and in fact ill-defined without a regularization procedure of some kind.

The authors are not aware of a non-perturbative regulator of the integral over three-metrics in (82) which preserves an exact diffeomorphism invariance. In the absence of such a regulator (which is also crucial for sorting out the operator-ordering issues [7]), we can only make some general remarks about the regularized form of $<\Delta q^{a}\left(x_{1}\right) \Delta q^{b}\left(x_{2}\right)>$. Inspection of (83) shows that $\left\langle\Delta q^{a}\left(x_{1}\right) \Delta q^{b}\left(x_{2}\right)>=0\right.$ for $x_{1} \neq x_{2}$; we also see that $\left(\mathcal{G}_{a b}^{E}\right)_{0} \Delta q^{a}(x) \Delta q^{b}(x)$ transforms like a scalar. One therefore expects the regulated expectation value to go like

$$
<\Delta q^{a}\left(x_{1}\right) \Delta q^{b}\left(x_{2}\right)>_{r e g} \approx \frac{\epsilon \hbar \kappa^{2}}{v}\left(\mathcal{G}^{E a b} \frac{1}{\sqrt{g}}\right)_{0} \delta^{3}\left(x_{1}-x_{2}\right)
$$

where $v$ is a scalar quantity with dimensions of volume, $v \rightarrow 0$ as the regulator is removed. A very important issue is whether $v$ has some dependence on the 3-metric $\left(g_{i j}\right)_{0}$, and this depends on the properties of the (unknown) regulator. If, as is the case with dynamical triangulation, there is a fixed short-distance cutoff $l$, then we expect that $v \approx l^{3}$, and the number of degrees of freedom changes with the volume of the manifold. If, on the other hand, the number of degrees of freedom $N_{p}$ is fixed (as in the Regge lattice), then the volume per degree of freedom changes with the volume of the manifold. In the latter case, it is reasonable to expect that

$$
<\Delta q^{a}\left(x_{1}\right) \Delta q^{b}\left(x_{2}\right)>_{r e g} \approx \epsilon \hbar \kappa^{2} \frac{\beta}{\mathbf{V}}\left(\mathcal{G}^{E a b} \frac{1}{\sqrt{g}}\right)_{0} \delta^{3}\left(x_{1}-x_{2}\right)
$$


where $\mathbf{V}$ is the volume of the 3 -manifold described by $\left(g_{i j}\right)_{0}$, and $\beta$ is proportional to the number of degrees of freedom in the regularization. A naive lattice regularization, replacing, e.g.

$$
\begin{aligned}
\Delta q^{a}(x) & \leftrightarrow \Delta q^{a}(n) \\
\int d^{3} x \sqrt{g} & \leftrightarrow \frac{\mathbf{V}}{N_{p}} \sum_{n=1}^{N_{p}} \\
\frac{\delta}{\delta q^{a}(x)} & \leftrightarrow \frac{N_{p} \sqrt{g(n)}}{\mathbf{V}} \frac{\partial}{\partial q^{a}(n)} \\
D q & \leftrightarrow \prod_{n} d^{D} q(n)
\end{aligned}
$$

does, in fact, lead to (85) (c.f. [4]), although of course such a regularization does not at all respect diffeomorphism invariance. We will assume that there exists some good regularization leading to (85), although our justification for this assumption is largely a posteriori.

Using eq. (85), the $T_{2}$ term (in (82)) contributing to the transfer matrix is easily evaluated; it is the only term which is important in computing the semiclassical limit. The other terms are operator-ordering contributions which, in the absence of an explicit regulator, we will ignore. The corresponding $Æ$ operator is

$$
" \mathbb{E} "=-\hbar^{2} \frac{\beta \kappa^{2}}{\mathbf{V}} \int d^{3} x U^{-1} G^{a b} \frac{\delta^{2}}{\delta q^{a} \delta q^{b}}
$$

where " $\mathbb{E} "$ is in quotes to emphasize that this operator, by itself, does not yield the correct equations of motion in the classical limit. This was to be expected, because $Æ$ has been derived by setting the shift functions $N_{i}=0$, a step which requires additional constraints on the physical states. Now note that because of the spatial volume denominator in (87), we can write the evolution equation (36) as

$$
\int d^{3} x Q_{x} \Psi=0
$$

where

$$
Q_{x}=-\hbar^{2} \kappa^{2} U^{-1} G^{a b} \frac{\delta^{2}}{\delta q^{a} \delta q^{b}}-i \hbar \sqrt{g} \frac{\partial}{\partial \tau}
$$

and where we have absorbed the constant $\beta$ in " $Æ$ " into a rescaling of the evolution parameter $\tau$ in eq. (36). The extra constraints which need to be imposed on the physical states, which then generate the usual constraint algebra of general relativity, are simply

$$
Q_{x} \Psi=0
$$

at every point $x$. To show this, consider an arbitrary solution of the evolution equation

$$
\Psi(q, \tau)=\sum_{\mathcal{E}} a_{\mathcal{E}} e^{i \mathcal{E} \tau / \hbar} \Phi_{\mathcal{E}}(q)
$$


Since the $a_{\mathcal{E}}$ are arbitrary, the Q-constraint (90) requires that for each stationary state

$$
\mathcal{H}_{x}^{\mathcal{E}} \Phi_{\mathcal{E}} \equiv\left\{-\hbar^{2} \frac{\kappa^{2}}{\sqrt{\mathcal{E}}} G^{a b} \frac{\delta^{2}}{\delta q^{a} \delta q^{b}}+\sqrt{\mathcal{E}} \sqrt{g} U\right\} \Phi_{\mathcal{E}}=0
$$

But this is simply the Wheeler-de Witt equation for the action $S_{\mathcal{E}}$ of eq. (26)! Moreover, the constraints $\mathcal{H}_{x}^{\mathcal{E}} \Phi_{\mathcal{E}}=0$ imply, via the Moncrief-Teitelboim interconnection theorem [9], that the supermomentum constraints

$$
\mathcal{H}_{x}^{i} \Phi_{\mathcal{E}}=0
$$

are satisfied as well. In this way, we find that the stationary states $\left\{\Phi_{\mathcal{E}}\right\}$ satisfy the usual constraint algebra of general relativity, given by the action $S_{\mathcal{E}}$. The Hilbert space of all physical states is spanned by the stationary states, with all possible values of $\mathcal{E}$.

Now multiplying both sides of (90) by $N U$, where $N$ is an arbitrary function, integrating over space, and applying the supermomentum constraint (93), we have in place of (87)

$$
\begin{aligned}
i \hbar \partial_{\tau} \Psi & =\left[\frac{1}{\int d^{3} x \sqrt{g} N U} \kappa^{2} \int d^{3} x N G^{a b}\left(-\hbar^{2} \frac{\delta^{2}}{\delta q^{a} \delta q^{b}}\right)\right] \Psi \\
& =\frac{1}{m_{P}} \int d^{3} x\left[-\hbar^{2} \tilde{N} \kappa^{2} G^{a b} \frac{\delta^{2}}{\delta q^{a} \delta q^{b}}+N_{i} \mathcal{H}_{x}^{i}\right] \Psi \\
& =Æ \Psi
\end{aligned}
$$

where

$$
Æ=\frac{1}{m_{P}} \int d^{3} x\left[-\hbar^{2} \tilde{N} \kappa^{2} G^{a b} \frac{\delta^{2}}{\delta q^{a} \delta q^{b}}+N_{i} \mathcal{H}_{x}^{i}\right]
$$

is the operator form of the Kinetic/Potential ratio (29) introduced in the previous section, with

$$
\tilde{N}(x) \equiv m_{P} \frac{N(x)}{\int d^{3} x^{\prime} \sqrt{g} N U(q)}
$$

It should be remembered that certain operator-ordering contributions to (94), coming from the $T_{0}$ and $T_{1}$ terms in (82) have been dropped. However, operator ordering terms are always $O(\hbar)$, and will not affect the correspondence of the $Æ$ operator to the Kinetic/Potential ratio (29) in the classical limit. From the fact that physical states are independent of $N$ and $N_{i}$

$$
\frac{\delta}{\delta N(x)} \circledast \Psi=\frac{\delta}{\delta N_{i}(x)} \circledast \Psi=0
$$

we find, as in the previous section, the generalized constraints

$$
\begin{aligned}
\left\{\left[\kappa^{2} G^{a b} p_{a} p_{b}\right]_{x}-[\sqrt{g} U]_{x} \mathbb{E}\right\} \Psi_{\text {phys }} & =0 \\
\mathcal{H}_{x}^{i} \Psi_{\text {phys }} & =0
\end{aligned}
$$


The closure of these generalized constraints under commutation depends only on the assumed closure of the standard constraints (42), for any fixed value of the parameter $\mathcal{E}$.

From the Schrödinger evolution equation (36), we have

$$
\begin{aligned}
\partial_{\tau}<q^{a}>= & \frac{i}{\hbar}<\left[\Subset, q^{a}\right]>=<\frac{\partial Æ}{\partial p_{a}}>+ \text { op.-ordering terms } \\
= & \frac{1}{m_{P}}<\int d^{3} x^{\prime}\left[\tilde{N}\left(x^{\prime}\right) \frac{\delta}{\delta p_{a}(x)}\left(\kappa^{2} G^{c d} p_{c} p_{d}\right)_{x^{\prime}}+N_{i}\left(x^{\prime}\right) \frac{\delta}{\delta p_{a}(x)} \mathcal{H}_{x^{\prime}}^{i}\right]> \\
& \quad+\text { op.-ordering terms } \\
\partial_{\tau}<p_{a}>= & \frac{i}{\hbar}<\left[Æ, p_{a}\right]>=<-\frac{\partial Æ}{\partial q^{a}}>+ \text { op.-ordering terms } \\
= & -<\frac{1}{m_{P}} \int d^{3} x^{\prime}\left[\tilde{N}\left(x^{\prime}\right) \frac{\delta}{\delta q^{a}(x)}\left(\kappa^{2} G^{c d} p_{c} p_{d}\right)_{x^{\prime}}\right. \\
& \left.-\tilde{N}\left(x^{\prime}\right) \notin \frac{\delta}{\delta q^{a}(x)}(\sqrt{g} U)_{x^{\prime}}+N_{i}\left(x^{\prime}\right) \frac{\delta}{\delta q^{a}(x)} \mathcal{H}_{x^{\prime}}^{i}\right]> \\
& + \text { op.-ordering terms }
\end{aligned}
$$

Equation (99) is the Ehrenfest principle obtained from our transfer matrix formalism. Removing the " $<>$ " brackets, replacing $Æ$ by a constant parameter $-\mathcal{E}$, and dropping operator-ordering terms, eq. (98) and (99) become the classical equations of motion (32).

\section{WKB, Time, and Many-Fingered Time}

In classical general relativity, the split of spacetime into space + time can be accomplished in infinitely many ways, which are distinguished by a choice of lapse and shift functions. The geometrical meaning of a time parameter $t$, in the classical equations of motion

$$
\begin{aligned}
\partial_{t} Q & =\{Q, H\} \\
& =\int d^{3} x\left[N(x)\left\{Q, \mathcal{H}_{x}\right\}+N_{i}(x)\left\{Q, \mathcal{H}_{x}^{i}\right\}\right]
\end{aligned}
$$

is specified by $N(x)$ and $N_{i}(x)$. This is also the case for the Æ-evolution equation

$$
\begin{aligned}
\partial_{\tau} Q & =\left\{Q, \circledast\left(p, q, N, N_{i}\right)\right\} \\
& =\frac{1}{m_{P}} \int d^{3} x\left[\sqrt{\mathcal{E}} \tilde{N}(x)\left\{Q, \mathcal{H}_{x}^{\mathcal{E}}\right\}+N_{i}(x)\left\{Q, \mathcal{H}_{x}^{i}\right\}\right]
\end{aligned}
$$

except that in the latter case the lapse $\tilde{N}$ has been normalized to satisfy the condition (33). To see what this restriction means for the evolution parameter $\tau$, let us consider 
Hamilton's principal function $S_{H P}^{\mathcal{E}}\left[q, q^{\prime}\right]$ satisfying the Hamilton-Jacobi equations and constraints

$$
\begin{aligned}
\kappa^{2} G_{a b} \frac{\delta S_{H P}^{\mathcal{E}}}{\delta q^{a}} \frac{\delta S_{H P}^{\mathcal{E}}}{\delta q^{b}}+\mathcal{E} \sqrt{g} U & =0 \\
\mathcal{H}^{i}\left[p_{a}=\frac{\delta S_{H P}^{\mathcal{E}}}{\delta q^{a}}\right] & =0
\end{aligned}
$$

and

$$
\begin{aligned}
\kappa^{2} G_{a b} \frac{\delta S_{H P}^{\mathcal{E}}}{\delta q^{\prime a}} \frac{\delta S_{H P}^{\mathcal{E}}}{\delta q^{\prime b}}+\mathcal{E} \sqrt{g} U & =0 \\
\mathcal{H}^{i}\left[p_{a}^{\prime}=-\frac{\delta S_{H P}^{\mathcal{E}}}{\delta q^{\prime a}}\right] & =0
\end{aligned}
$$

As usual, the principal function $S_{H P}^{\mathcal{E}}$ defined in this way has the interpretation of being the action of the classical trajectory in superspace, connecting configurations $q^{a}(x)$ and $q^{a}(x)$. Then consider the variation of $S_{H P}^{\mathcal{E}}$ in time $\tau$ according to (101)

$$
\begin{aligned}
\Delta S_{H P}^{\mathcal{E}} & =\frac{d S_{H P}^{\mathcal{E}}}{d \tau} \Delta \tau=\left\{S_{H P}^{\mathcal{E}}, \mathbb{E}\right\} \tau \\
& =\int d^{3} x \frac{\delta S_{H P}^{\mathcal{E}}}{\delta q^{a}} \frac{\delta \mathbb{E}}{\delta p_{a}} \Delta \tau \\
& =\frac{1}{m_{P}} \int d^{3} x \frac{\delta S_{H P}^{\mathcal{E}}}{\delta q^{a}}\left\{2 \kappa^{2} \tilde{N} G^{a b} \frac{\delta S_{H P}^{\mathcal{E}}}{\delta q^{b}}+\frac{\delta}{\delta p_{a}(x)} \int d^{3} x^{\prime} N_{i} \mathcal{H}_{x^{\prime}}^{i}\right\} \Delta \tau \\
& =\frac{1}{m_{P}} \int d^{3} x\left\{2 \kappa^{2} \tilde{N} G^{a b} \frac{\delta S_{H P}^{\mathcal{E}}}{\delta q^{a}} \frac{\delta S_{H P}^{\mathcal{E}}}{\delta q^{b}}+N_{i} \mathcal{H}_{x}^{i}\left[p_{a}=\frac{\left.\delta S_{H P}^{\mathcal{E}}\right]}{\delta q^{a}}\right]\right\} \tau \\
& =2 \notin\left[p_{a}=\frac{\delta S_{H P}^{\mathcal{E}}}{\delta q^{a}}\right] \Delta \tau \\
& =-2 \mathcal{E} \Delta \tau
\end{aligned}
$$

What eq. (104) establishes is that, in evolving classically from configuration $q^{\prime}$ to $q$ in time $\Delta \tau$ according to (101), the action $S_{H P}^{\mathcal{E}}\left[q, q^{\prime}\right]$ is proportional to $\Delta \tau$ regardless of the choice of $N, N_{i}$. In other words, while the particular configuration $q$ reached after $\Delta \tau$ does depend on $N, N_{i}$, the increment of action $\Delta S$ does not. This situation is indicated schematically in Figure 1. Of course, the restriction $\Delta \tau \propto \Delta S_{H P}^{\mathcal{E}}$ is not a restriction on hypersurfaces; $Q[q, p]$ can be computed from (101) on any spacelike hypersurface of the classical manifold, by choosing an appropriate $N, N_{i}$. For this reason, the $Æ$-evolution equation (101) is just as informative as the traditional evolution equation (100).

The quantum equation of motion for an observable $Q$, in our formalism, is

$$
\left.\partial_{\tau}<Q>=-\frac{i}{\hbar}<\Psi(q, \tau)\right]|[Q, \circledast]| \Psi(q, \tau)>
$$


The correspondence between (105) and (101) in the semiclassical limit can be studied using the WKB approximation. In this approximation, to leading order in $\hbar$, a solution of $Æ \Phi=-\mathcal{E} \Phi$ and all other constraints (42) has the form

$$
\Phi_{\mathcal{E}, q^{\prime}}(q)=\exp \left[i \sqrt{\mathcal{E}} S_{H P}\left[q, q^{\prime}\right] / \hbar\right]
$$

where

$$
S_{H P} \equiv \frac{S_{H P}^{\mathcal{E}}}{\sqrt{\mathcal{E}}}
$$

is Hamilton's principal function for $\mathcal{E}=1$. Of course, eq. (106) is only valid away from caustics, in the classically allowed region. We can now write a general $\tau$-dependent WKB solution as a wavepacket of the form

$$
\begin{aligned}
\Psi(q, \tau) & =\int d \mathcal{E} D q^{\prime} F\left[\mathcal{E}, q^{\prime}\right] \exp [i \tilde{S}] \\
& =\int d \mathcal{E} D q^{\prime} F\left[\mathcal{E}, q^{\prime}\right] \exp \left[i\left\{\mathcal{E} \tau+\sqrt{\mathcal{E}} S_{H P}\left[q, q^{\prime}\right]+\theta\left[q^{\prime}\right]\right\} / \hbar\right]
\end{aligned}
$$

where $F\left[\mathcal{E}, q^{\prime}\right]$ and $\theta\left(q^{\prime}\right)$ are real-valued. To make contact with classical physics, suppose that $F$ is sharply peaked around some value $\mathcal{E}_{0}$ and configuration $q_{0}^{a}(x)$, and define

$$
p_{0 a}(x) \equiv\left(\frac{\delta \theta\left[q^{\prime}\right]}{\delta q^{\prime a}(x)}\right)_{q^{\prime}=q_{0}}
$$

Then, according to the principle of constructive interference [5], the wavefunction $\Psi(q, \tau)$ will be peaked around configurations $q^{a}(x)$ such that the phase $\tilde{S}$ in (108) is stationary w.r.t. small variations of the parameters $\mathcal{E}, q^{\prime}$ around $\mathcal{E}_{0}, q_{0}^{a}(x)$. In other words, $q^{a}(x)$ satifies

$$
\begin{aligned}
& \left(\frac{\partial \tilde{S}}{\partial \mathcal{E}}\right)_{\mathcal{E}=\mathcal{E}_{0}}=0 \quad \Rightarrow \quad \tau=-\frac{1}{2 \sqrt{\mathcal{E}_{0}}} S_{H P}\left[q, q^{\prime}\right] \\
& \left(\frac{\delta \tilde{S}}{\delta q^{\prime a}}\right)_{q^{\prime}=q_{0}}=0 \quad \Rightarrow \quad p_{0 a}=-\sqrt{\mathcal{E}_{0}}\left(\frac{\delta S_{H P}}{\delta q^{\prime a}}\right)_{q^{\prime}=q_{0}}
\end{aligned}
$$

The principle of constructive interference is the standard way to make the connection between WKB wavefunctions and the classical equations of motion [5], [6]. Equations (110), with $S_{H P}$ satisfying the Hamiltonian-Jacobi equations (102) and (103), are sufficient to specify a classical solution $q^{a}(x, \tau)$ beginning from an initial configuration $q^{a}(x, 0)=q_{0}^{a}(x)$, and initial momenta $p_{0 a}(x)$ satisfying the appropriate constraint equations (103).

The first of the equations in (110) tells us that, for WKB wavefunctions of the form (108), the "wavefunction of the Universe" is not peaked around a single 3-manifold at time $\tau$, but is instead peaked at configurations (3-manifold + fields) $q^{a}(x, \tau)$ in 
superspace such that the classical action between the initial configuration $q_{0}$ and $q^{a}$ is a constant, i.e.

$$
\begin{aligned}
\tau & =-\frac{1}{2 \sqrt{\mathcal{E}_{0}}} S_{H P}\left[q, q_{0}\right] \\
& =-\frac{1}{2 \mathcal{E}_{0}} S_{H P}^{\mathcal{E}_{0}}\left[q, q_{0}\right]
\end{aligned}
$$

Therefore, even in the classical limit, $\tau$ does not in general single out a particular 3manifold and set of fields, obeying (111). The second equation in (110) is equivalent to the dynamical equations of the classical theory, and ensures that the wavefunction is peaked only at configurations $q^{a}$ obtained from spacelike slices of the classical solution determined by an initial $\left\{q_{0}^{a}, p_{0 a}\right\}$.

The situation for $F\left[\mathcal{E}, q^{\prime}\right]$ concentrated at some $\mathcal{E}_{0}$ and $q_{0}$ is summarized in Fig. 2. At a given value of the evolution parameter $\tau$, the wavefunction of the Universe is peaked at a whole class of configurations, which correspond to spacelike slices of the classical, 4-dimensional solution, satisfying the restriction (111).

Now after a measurement of some observable, it is expected that the Universe should be left in an eigenstate, or approximate eigenstate, of that observable immediately after the measurement. This is a great difficulty for the standard formulation of canonical quantum gravity, since an eigenstate of, e.g., 3-geometry is not a solution of the Wheeler-de Witt equation, and therefore not a physical state. In our formulation, the situation is more favorable. Imagine that some measurement were performed which determined the values of the observables $\left\{q^{a}, p_{a}\right\}$, or some subset of these observables (modulo three-dimensional diffeomorphisms) up to the limits imposed by the $\Delta q \Delta p$ uncertainty principle. Immediately after the measurement, the Universe would be left in a state of the form (108) with $\tau=0$, and $q_{0}=q_{\text {observed }}$ (modulo three-dimensional diffeomorphisms). This means that, at $\tau=0$, the wavefunction of the Universe would be peaked around configurations $q^{a}$ satisfying

$$
S_{H P}\left[q, q_{\text {observed }}\right]=0
$$

Since: (i) the action $S_{H P}\left[q, q^{\prime}\right]$ of a classical solution bounded by $q$ and $q^{\prime}$ is only well-defined if $q^{\prime}$ is a Cauchy surface for $q$ (i.e. if $q$ and $q^{\prime}$ do not intersect on the 4-dimensional solution manifold); and noting that (ii) the classical action is monotonic in the evolution parameter (see eq. (104)), away from caustics/turning points; eq. (112) implies that the wavefunction at $\tau=0$ is peaked only at configurations which are equivalent, up to three-dimensional diffeomorphisms, to

$$
q^{a}(x)=q_{\text {observed }}^{a}(x)
$$


In other words, there exist physical states which are at least approximate eigenstates of quantities such as 3-geometry, and this is made possible by a dispersion in the value of $\mathcal{E}$.

\section{- The "Energy-Time" Uncertainty Relation}

As discussed at length in section 2 , the quantity $\mathcal{E}$ is classically irrelevant: it does not appear in the Euler-Lagrange equations, and therefore cannot be determined from a classical orbit in superspace. In fact, it is easy to see that an uncertainty in $\mathcal{E}$ can be interpreted as an uncertainty in the effective value of Planck's constant. This is because in the constraint equation (92) $\mathcal{E}$ can simply be absorbed into a redefinition of $\hbar$, i.e.

$$
\left\{-\hbar_{e f f}^{2} \kappa^{2} G^{a b} \frac{\delta^{2}}{\delta q^{a} \delta q^{b}}+\sqrt{g} U\right\} \Phi_{\mathcal{E}}=0
$$

where

$$
\hbar_{e f f}=\frac{\hbar}{\sqrt{\mathcal{E}}}
$$

Whatever the correct operator-ordering may be, the absorbtion of $\mathcal{E}$ into $\hbar$ is always possible.

We would like to have a quantitative estimate relating the uncertainty in $\mathcal{E}$ (or $\hbar_{e f f}$ ) to the spread of wavefunctions along a classical trajectory (in superspace) around $q_{\text {observed }}^{a}(x)$, leading to an analog of the time-energy uncertainty relation in ordinary quantum mechanics. The discussion below may serve to illustrate some of the issues and ambiguities involved in that estimate.

Consider a WKB wavepacket with $F\left[\mathcal{E}, q^{\prime}\right]$ of the form

$$
F\left[\mathcal{E}, q^{\prime}\right]=\frac{1}{2 \rho} f\left[q^{\prime}\right] \exp \left[-\frac{\left(\rho-\rho_{0}\right)^{2}}{(\Delta \rho)^{2}}\right]
$$

where $\rho \equiv \sqrt{\mathcal{E}}$. Then, integrating over $\mathcal{E}$ in (108) we have, at $\tau=0$,

$$
\Psi(q, 0)=\int D q^{\prime} f\left[q^{\prime}\right] e^{i\left(\theta\left[q^{\prime}\right]+\rho_{0} S_{H P}\left[q, q^{\prime}\right]\right) / \hbar} \exp \left[-(\Delta \rho)^{2} S_{H P}^{2}\left[q, q^{\prime}\right] / 4 \hbar^{2}\right]
$$

Assuming, as before, that $f\left[q^{\prime}\right]$ is peaked (modulo diffeomorphisms) at some configuration $q_{0}^{a}(x)$, the integrand has a stationary phase along the classical manifold statisfying the second of equations (110). Consider a configuration $q_{c l}$ along the classical manifold. Away from $q_{c l}=q_{0}$, there will be a suppression factor in the wavefunction

$$
\begin{aligned}
\left|\Psi\left(q_{c l}, 0\right)\right|^{2} & \sim \exp \left[-(\Delta \rho)^{2} S_{H P}^{2}\left[q_{c l}, q_{0}\right] / 2 \hbar^{2}\right] \\
& \sim \exp \left[-\left(\frac{\Delta \mathcal{E}}{\mathcal{E}_{0}} S_{H P}^{\mathcal{E}_{0}}\left(q_{c l}, q_{0}\right)\right)^{2} / 8 \hbar^{2}\right]
\end{aligned}
$$


If we ask only for the spread of the wavefunction along the classical manifold, then clearly

$$
\frac{\Delta \mathcal{E}}{\mathcal{E}_{0}} S_{H P}^{\mathcal{E}_{0}}\left(q_{c l}, q_{0}\right) \sim \hbar
$$

or, taking into account eq. (104),

$$
\Delta \mathcal{E} \Delta \tau \sim \hbar
$$

which has the form of the usual energy-time uncertainty relation. However, since $\tau$ is just an evolution parameter rather than, e.g., a proper time interval, this relation is not very informative.

Let us consider the probability density $P(\Delta S)$ for being at any configuration $q_{c l}$ such that

$$
\Delta S=S_{H P}\left[q_{c l}, q_{0}\right]
$$

In that case, the measure $\mu(\Delta S)$ of such configurations along the classical solution manifold becomes important:

$$
P(\Delta S) \sim \mu(\Delta S) \exp \left[-\left(\frac{\Delta \mathcal{E}}{\sqrt{\mathcal{E}_{0}}} \Delta S\right)^{2} / 8 \hbar^{2}\right]
$$

In the absence of a regulator for general relativity, we don't really know the measure $\mu(\Delta S)$. But let us imagine that it has some simple power-law dependence on the number of degrees of freedom $N_{p}$

$$
\mu(\Delta S) \sim(\Delta S)^{\alpha N_{p}}
$$

where $\alpha$ is a constant of $O(1)$. In that case, $P(\Delta S)$ would be a maximum at

$$
\left(\frac{\Delta \mathcal{E}}{\mathcal{E}_{0}} \Delta S\right)^{2}=4 \alpha \hbar_{e f f}^{2} N_{p}
$$

To relate $\Delta S$ to an increment of proper time $\Delta s$ along the classical manifold, let us consider a matter-dominated Friedman universe, in which

$$
\Delta S \sim M \Delta s
$$

where $M$ is the total mass, and $\Delta s$ is the average increment of proper time between $q_{0}$ and $q_{c l}$ in a synchronous coordinate system. Then we have

$$
\left(\frac{\Delta \mathcal{E}}{\mathcal{E}_{0}} \Delta s\right)^{2} \sim \alpha \hbar_{e f f}^{2} \frac{N_{p}}{M^{2}}
$$


Let $V$ be the volume and $\rho_{m}$ the density of matter in the Universe, and denote by $v_{0}$ the volume per degree of freedom. Then

$$
\frac{\Delta \mathcal{E}}{\mathcal{E}_{0}} \Delta s \sim \frac{\hbar_{e f f}}{\rho_{m}} \sqrt{\frac{\alpha}{v_{0} V}}
$$

or, equivalently,

$$
\frac{\Delta \hbar_{e f f}}{\hbar_{e f f}} \Delta s \sim \hbar_{e f f} \sqrt{\frac{\alpha}{\rho_{m}^{2} v_{0} V}}
$$

which is the analog, in our formulation, of the energy-time uncertainty relation of non-parametrized theories.

It is difficult to assign a reliable number to the right hand side of expression (128), even assuming the validity of eq. (123) as a measure of configurations, which was used in the derivation. The mass density $\rho_{m}$ can be taken as, roughly, the critical density for closing the Universe $\left(\sim 10^{-26} \mathrm{~kg} / \mathrm{m}^{3}\right)$, and $v_{0}$, assuming it is different from zero, is presumably the Planck volume $\left(\sim 10^{-105} \mathrm{~m}^{3}\right)$. But there is still tremendous uncertainty in the volume $V$ of the Universe. One could even speculate that, in an appropriate continuum limit of regularized quantum gravity, $v_{0} \rightarrow 0$ and $V \rightarrow \infty$ such that $v_{0} V$ tends to a finite constant. In any case, while it appears that the transfer matrix formulation must generate some uncertainty relation between proper time and the fractional uncertainty in Planck's constant, we are so far unable to say anything quantitative.

\section{Non-Stationary States in Minisuperspace}

We will now illustrate the formalism developed above in the simplest context possible: the quantum mechanical time-evolution of a homogeneous, isotropic Friedmann universe filled with a relativistic perfect fluid. In this toy model it is possible to "do everything"; i.e. to find the integration measure and operator ordering, to solve for the spectrum and exact eigenstates of the $Æ$ evolution operator, to form wavepackets with a conserved norm, and to track the evolution of such "wavefunctions of the Universe" through collapse towards the singularity, "bounce", expansion, and recollapse.

The Friedmann universe is described by the metric

$$
d s^{2}=\sigma^{2}\left[-N^{2} d t^{2}+a^{2} d \Omega_{3}^{2}\right]
$$

where $N$ and $a$ are the lapse and scale factors and $\sigma^{2}=\frac{2 G_{N}}{3 \pi}$. The perfect fluid content of this homogeneous and isotropic universe is specified by the energy-momentum tensor

$$
T_{\mu \nu}=(\rho+p) U_{\mu} U_{\nu}+p g_{\mu \nu}
$$


where $g_{\mu \nu}$ is the Friedmann metric corresponding to the line element (129), $U_{\mu}=$ $(1,0,0,0)$ with $U_{\mu} U^{\mu}=-1$ is the four velocity of the fluid and $\rho$ and $p$ are, respectively, the energy density and pressure of the fluid.

For a perfect fluid described by the equation of state

$$
p=(\gamma-1) \rho
$$

the action for gravity and matter (with zero bare cosmological constant) which is consistent with the definition of the energy tensor (130) (see, e.g., [10]) can be written as

$$
S_{b m}=\frac{1}{2} \int d t\left[-\frac{a \dot{a}^{2}}{N}+N a-N a_{0}^{3 \gamma-2} a^{3(1-\gamma)}\right]
$$

Classically, these models represent, for $\gamma>2 / 3$, a universe which expands up to a maximum radius $a_{0}$ and then recollapses towards the singularity at $a=0$ and, for $\gamma<2 / 3$, an inflationary universe expanding from the minimum radius $a_{0}$ (see, e.g., [13]) ${ }^{4}$

The perfect fluid does not introduce any extra dynamical field in this model, and it is easy to rewrite the action (132) in the Hamiltonian form corresponding to the time-parametrized theory of eq. (12). The only degrees of freedom are given by the scale factor $\left(q^{a}=a\right)$ and its conjugate momentum $p_{a}=-\frac{a \dot{a}}{N}$, and the Hamiltonian $\mathcal{H}$ is given by eq. (12) with

$$
\begin{aligned}
G_{a a} & =-a \\
V(a) & =\frac{a}{2}\left[\left(\frac{a}{a_{0}}\right)^{2-3 \gamma}-1\right]
\end{aligned}
$$

(and $m_{0}=1$ ). The 'superpotential' $V$ is positive definite in the classically allowed region $a<a_{0}$ for $\gamma>2 / 3\left(a>a_{0}\right.$ for $\left.\gamma<2 / 3\right)$, and it changes sign at the classical turning point $a=a_{0}$.

Following the discussion of section 3, we can immediately write the expression for the evolution operator $Æ$ in the 1-d minisuperspace (see eq. (67)) as

$$
Æ=-\frac{\hbar^{2}}{|\mathcal{G}|^{1 / 2}} \frac{\partial}{\partial a}|\mathcal{G}|^{1 / 2} \mathcal{G}^{a a} \frac{\partial}{\partial a}
$$

In this case there is no operator ordering term involving $\mathcal{R}$ since, obviously, a one dimensional superspace has vanishing scalar curvature $\mathcal{R}=0$. More explicitly, using $\mathcal{G}_{a a}=\mathcal{G}=-2 a V(a)$ and eq. (133), we can write

$$
\rightleftarrows=\hbar^{2}[2 a|V|]^{-1 / 2} \frac{\partial}{\partial a} \frac{[2 a|V|]^{1 / 2}}{2 a V} \frac{\partial}{\partial a}=
$$

\footnotetext{
${ }^{4}$ Also note that we must have $\gamma \leq 2$ for the sound wave velocity of the fluid to be less than the speed of light.
} 


$$
=\frac{\hbar^{2}}{2 a V}\left[\frac{\partial^{2}}{\partial a^{2}}-\frac{1}{2 a}\left(1+\frac{a}{V} \frac{\partial V}{\partial a}\right) \frac{\partial}{\partial a}+\delta\left(a-a_{0}\right) \operatorname{sign}\left(V \frac{\partial V}{\partial a}\right) \frac{\partial}{\partial a}\right]
$$

The problem is now to solve for all the stationary states

$$
Æ \Phi_{\mathcal{E}}[a]=-\mathcal{E} \Phi_{\mathcal{E}}[a]
$$

each of which is a solution of a Wheeler-de Witt equation with a particular operatorordering, and an effective value of Planck's constant which depends on $\mathcal{E}$ (see eq. (115)). With these solutions in hand, we can then construct non-stationary states as a linear superposition

$$
\Psi[a, \tau]=\sum_{\mathcal{E}} a_{\mathcal{E}} \Phi_{\mathcal{E}}[a] e^{i \mathcal{E} \tau / \hbar}
$$

Since the evolution operator $Æ$ of $(135)$ is very singular at the classical turning point $a=a_{0}$ (the second line of eq. (135) does not even make sense, since the product of two distributions is not defined) we shall consider the regions where $V>0$ and $V<0$ separately, and then impose some appropriate junction condition at the classical turning point $a=a_{0}$.

Let us first consider the region $V>0$. In this region we change from the coordinate $a$ to the 'tortoise' coordinate

$$
a_{+} \doteq \pm \int_{a_{0}}^{a} d \bar{a}[2 V \bar{a}]^{1 / 2}= \pm \int_{a_{0}}^{a} d \bar{a} \bar{a}\left[\left(\frac{\bar{a}}{a_{0}}\right)^{2-3 \gamma}-1\right]^{1 / 2}
$$

where the plus sign is for the case $\gamma<2 / 3$ and the minus sign for $\gamma>2 / 3$. The coordinate $a_{+}$is chosen such as to start from zero at $a=a_{0}$ and to be semi-positive definite, monotonically growing to infinity as $a>a_{0} \rightarrow \infty(\gamma<2 / 3)$ or to the maximum $a_{+M}>0$ as $a \rightarrow 0(\gamma>2 / 3):$

$$
\begin{array}{llcc}
a_{+} \in[0, \infty), \quad a>a_{0}, & \gamma<2 / 3 \\
a_{+} \in\left[0, a_{+M}\right], \quad a<a_{0}, & \gamma>2 / 3 \\
a_{+} \geq 0, \quad a_{+}\left(a_{0}\right)=0, & a_{+}(0)=a_{+M}
\end{array}
$$

With this choice, the eigenvalue problem for the evolution operator simply becomes

$$
\hbar^{2} \frac{\partial^{2}}{\partial a_{+}^{2}} \Phi_{\mathcal{E}}^{+}\left[a_{+}\right]=-\mathcal{E} \Phi_{\mathcal{E}}^{+}\left[a_{+}\right]
$$

which is the Schrödinger problem for the motion of a free particle with energy $\mathcal{E}$. Assuming from now on that $\mathcal{E}>0$. p the general solution of eq. (140) in the region $V>0$ is clearly a combination of plane waves

$$
\Phi_{\mathcal{E}}^{+}\left[a_{+}\right]=A e^{i \sqrt{\mathcal{E}} a_{+} / \hbar}+B e^{-i \sqrt{\mathcal{E}} a_{+} / \hbar}
$$

\footnotetext{
${ }^{5}$ The choice $\mathcal{E}>0$ is required in order to have a real-valued Planck's constant.
} 
Similarly, in the region $V<0$, we transform to the 'tortoise' coordinate

$$
a_{-} \doteq \pm \int_{a_{0}}^{a} d \bar{a}[-2 V \bar{a}]^{1 / 2}= \pm \int_{a_{0}}^{a} d \bar{a} \bar{a}\left[1-\left(\frac{\bar{a}}{a_{0}}\right)^{2-3 \gamma}\right]^{1 / 2}
$$

where, again, the plus sign is for the case $\gamma<2 / 3$ and the minus sign for $\gamma>2 / 3$. The coordinate $a_{-}$is zero at $a=a_{0}$ and negative semi-definite, monotonically growing to minus infinity as $a>a_{0} \rightarrow \infty(\gamma>2 / 3)$ or to the minimum $a_{-m}<0$ as $a \rightarrow 0$ $(\gamma<2 / 3)$ :

$$
\begin{array}{lll}
a_{-} \in\left[a_{-m}, 0\right] \quad, \quad a<a_{0}, & \gamma<2 / 3 \\
a_{-} \in(-\infty, 0] \quad, \quad a>a_{0}, & \gamma>2 / 3 \\
a_{-} \leq 0, \quad a_{-}\left(a_{0}\right)=0, & a_{-}(0)=a_{-m}
\end{array}
$$

In this case, eq. (136) becomes

$$
\hbar^{2} \frac{\partial^{2}}{\partial a_{-}^{2}} \Phi_{\mathcal{E}}^{-}\left[a_{-}\right]=\mathcal{E} \Phi_{\mathcal{E}}^{-}\left[a_{-}\right]
$$

with solutions in the (classically forbidden) $a_{-}$region

$$
\Phi_{\mathcal{E}}^{-}\left[a_{-}\right]=C e^{\sqrt{\mathcal{E}} a_{-} / \hbar}+D e^{-\sqrt{\mathcal{E}} a_{-} / \hbar}
$$

Let us now build the general solution of the original eigenvalue problem (136) in the whole range $a \in[0, \infty)$.

First, we must impose a junction condition at the turning point $a_{+}=a_{-}=0$ $\left(a=a_{0}\right)$. We multiply both sides of eq. (136) by $[2 a|V|]^{1 / 2}$ and, using eq. (135), we integrate around the turning point $a_{0}$. Assuming a reasonably smooth behaviour of $\Phi_{\mathcal{E}}$ at $a_{0}$, we find that

$$
\lim _{\delta \rightarrow 0} \int_{a_{0}-\delta}^{a_{0}+\delta} d a \frac{\partial}{\partial a} \frac{[2 a|V|]^{1 / 2}}{2 a V} \frac{\partial}{\partial a} \Phi_{\mathcal{E}}=-\lim _{\delta \rightarrow 0} \int_{a_{0}-\delta}^{a_{0}+\delta} d a[2 a|V|]^{1 / 2} \frac{\mathcal{E}}{\hbar^{2}} \Phi_{\mathcal{E}}=0
$$

Evaluating the integral on the left hand side, the joining condition on first derivatives of $\Phi_{\mathcal{E}}$ in the a coordinate can be rewritten as

$$
\left.\frac{|V|^{1 / 2}}{V} \frac{\partial \Phi_{\mathcal{E}}}{\partial a}\right|_{a_{0+}}=\left.\frac{|V|^{1 / 2}}{V} \frac{\partial \Phi_{\mathcal{E}}}{\partial a}\right|_{a_{0^{-}}}
$$

Both $\Phi_{\mathcal{E}}$ and its derivative must be continuous at $a=a_{0}$.

When we turn to $a_{ \pm}$coordinates, it is easy to see that first derivatives must be 'discontinuous', i.e. we have the junction conditions

$$
\begin{aligned}
\Phi_{\mathcal{E}}^{+}[0] & =\Phi_{\mathcal{E}}^{-}[0] \\
\left.\frac{\partial \Phi_{\mathcal{E}}^{ \pm}}{\partial a_{ \pm}}\right|_{a_{0^{+}}} & =-\left.\frac{\partial \Phi_{\mathcal{E}}^{\mp}}{\partial a_{\mp}}\right|_{a_{0^{-}}}
\end{aligned}
$$


where in the last equation upper signs refer to the case $\gamma<2 / 3$, and lower signs to the case $\gamma>2 / 3$.

Second, the evolution operator is defined on the 'half line' $a>0$, and therefore it is not essentially self-adjoint in $L^{2}[0, \infty)$ with the measure $\sqrt{|\mathcal{G}|}$. One can build, however, a one-parameter family of self-adjoint extensions (which guarantee norm conservation and unitarity) by appropriately choosing boundary conditions at the origin (see, e.g., [14). In the $a_{ \pm}$coordinates this is translated into the condition

$$
\left.\frac{\partial \Phi_{\mathcal{E}}^{ \pm}}{\partial a_{ \pm}}\right|_{a_{ \pm, m(M)}}=\left.\alpha \Phi_{\mathcal{E}}^{ \pm}\right|_{a_{ \pm, m(M)}}
$$

where $\alpha$ is an arbitrary real parameter with range $(-\infty, \infty)$. The lower signs (and index $m$ ) correspond to the case $\gamma<2 / 3$, while the upper signs (and index $M$ ) correspond to $\gamma>2 / 3$.

Self-adjointness of the evolution operator $\mathbb{E}$ automatically guarantees orthogonality of eigenfunctions, i.e.

$$
\begin{aligned}
\int_{0}^{\infty} d a \sqrt{|\mathcal{G}|} \Phi_{\mathcal{E}_{1}}^{*}[a] \Phi_{\mathcal{E}_{2}}[a] & =\int_{a_{-m}(-\infty)}^{0} d a_{-} \Phi_{\mathcal{E}_{1}^{-}}^{*}\left[a_{-}\right] \Phi_{\mathcal{E}_{2}}^{-}\left[a_{-}\right] \\
& +\int_{0}^{\infty\left(a_{+M}\right)} d a_{+} \Phi_{\mathcal{E}_{1}}^{+}\left[a_{+}\right] \Phi_{\mathcal{E}_{2}}^{+}\left[a_{+}\right]=\delta\left(\mathcal{E}_{1}, \mathcal{E}_{2}\right)
\end{aligned}
$$

where $\delta\left(\mathcal{E}_{1}, \mathcal{E}_{2}\right)$ is the Dirac delta function when the spectrum is continuous and the Kronecker delta for a discrete spectrum (and integration limits in parenthesis are for the case $\gamma>2 / 3$ ).

It is also possible to show that the system of eigenfunctions is complete, i.e., defining $\rho \doteq \sqrt{\mathcal{E}}$, that

$$
\sum_{n} \Phi_{\rho_{n}}^{*}[a] \Phi_{\rho_{n}}\left[a^{\prime}\right]=\frac{\delta\left(a-a^{\prime}\right)}{\sqrt{|\mathcal{G}|}}
$$

(where of course the sum is meant as an integral for a continuous spectrum).

We are now in the position to write the exact eigenfunctions for different $\gamma$ as a function of the scale factor $a$.

- $\gamma<2 / 3$

In the case $\gamma<2 / 3$, the spectrum is continuous and non degenerate, and from conditions (148)-(149) one finds, for a generic boundary condition at the origin $a=0$,

$$
\begin{aligned}
A & =\left[(1+i)+(1-i) \frac{(\sqrt{\mathcal{E}}-\hbar \alpha)}{(\sqrt{\mathcal{E}}+\hbar \alpha)} e^{2 \sqrt{\mathcal{E}} a_{-m} / \hbar}\right] \frac{C}{2} \\
B & =\left[(1-i)+(1+i) \frac{(\sqrt{\mathcal{E}}-\hbar \alpha)}{(\sqrt{\mathcal{E}}+\hbar \alpha)} e^{2 \sqrt{\mathcal{E}} a_{-m} / \hbar}\right] \frac{C}{2} \\
D & =\frac{(\sqrt{\mathcal{E}}-\hbar \alpha)}{(\sqrt{\mathcal{E}}+\hbar \alpha)} e^{2 \sqrt{\mathcal{E}} a_{-m} / \hbar} C
\end{aligned}
$$


For instance, in the case $\gamma=0$, which classically corresponds to a de Sitter universe expanding from the minimum radius $a_{0}$, the 'tortoise' coordinates derived from eqs. (138) and (142) are

$$
\begin{aligned}
a_{-} & =-\frac{a_{0}^{2}}{3}\left[1-\left(\frac{a}{a_{0}}\right)^{2}\right]^{3 / 2} \\
a_{+} & =\frac{a_{0}^{2}}{3}\left[\left(\frac{a}{a_{0}}\right)^{2}-1\right]^{3 / 2} \\
a_{-m} & =-\frac{a_{0}^{2}}{3}
\end{aligned}
$$

Moreover, if we choose boundary conditions at the origin such that $\Phi_{\mathcal{E}}(0)=0$ (or, equivalently, from eq. (149), such that $\alpha=\infty$ ), the eigenfunctions of $\mathbb{E}$ are

$$
\Phi_{\mathcal{E}}[a]=C\left\{e^{-\frac{\sqrt{\mathcal{E}} a_{0}^{2}}{3 \hbar}\left[1-\left(\frac{a}{a_{0}}\right)^{2}\right]^{3 / 2}}-e^{\frac{\sqrt{\mathcal{E}} a_{0}^{2}}{3 \hbar}\left[\left[1-\left(\frac{a}{a_{0}}\right)^{2}\right]^{3 / 2}-2\right]}\right\}
$$

for $a<a_{0}$ and

$$
\begin{aligned}
\Phi_{\mathcal{E}}[a] & =2 C e^{-\frac{\sqrt{\mathcal{E}} a_{0}^{2}}{3 \hbar}}\left\{\sinh \left[\frac{\sqrt{\mathcal{E}} a_{0}^{2}}{3 \hbar}\right] \cos \left[\frac{\sqrt{\mathcal{E}} a_{0}^{2}}{3 \hbar}\left[\left(\frac{a}{a_{0}}\right)^{2}-1\right]^{3 / 2}\right]\right. \\
& \left.-\cosh \left[\frac{\sqrt{\mathcal{E}} a_{0}^{2}}{3 \hbar}\right] \sin \left[\frac{\sqrt{\mathcal{E}} a_{0}^{2}}{3 \hbar}\left[\left(\frac{a}{a_{0}}\right)^{2}-1\right]^{3 / 2}\right]\right\}
\end{aligned}
$$

for $a>a_{0}$. With this choice of boundary conditions, the eigenfunction is real for all values of $a$.

- $\gamma>2 / 3$

The case $\gamma>2 / 3$ corresponds classically to a universe which expands from the singularity at $a=0$ to the maximum radius at $a=a_{0}$, and then collapses back towards the singularity. Since now the range of the 'tortoise' coordinate $a_{-}$is infinite (see eq. (143)), to ensure square integrability of the eigenfunctions (eq. (150)) clearly we must have $D=0$. Therefore, due to conditions (148) and (149), the spectrum is discrete and non degenerate.

Normalizing the eigenfunctions to one in the range $a \in[0, \infty)$, from eqs. (148) and (150) we have that

$$
A=\frac{(1+i)}{2} C
$$




$$
\begin{aligned}
B & =\frac{(1-i)}{2} C \\
C & =\left[\frac{2 \sqrt{\mathcal{E}}}{\hbar \cos \left(2 \sqrt{\mathcal{E}} a_{+M} / \hbar\right)+2 \sqrt{\mathcal{E}} a_{+M}}\right]^{1 / 2} e^{i \theta}
\end{aligned}
$$

where $\theta$ is an arbitrary phase.

Moreover, imposing a generic boundary condition at the origin according to eq. (149) gives an implicit expression for the discrete spectrum of $\mathcal{E}$, i.e.

$$
\tan \left(\sqrt{\mathcal{E}} a_{+M} / \hbar\right)=\frac{\hbar \alpha+\sqrt{\mathcal{E}}}{\hbar \alpha-\sqrt{\mathcal{E}}}
$$

For example, in the case of a pressureless, dust-dominated universe with $\gamma=1$, the explicit form of the 'tortoise' coordinates is

$$
\begin{aligned}
a_{-} & =\frac{a_{0}^{2}}{4}\left[\operatorname{arccosh}\left(\frac{a}{a_{0}}\right)^{1 / 2}+\left(1-\frac{2 a}{a_{0}}\right)\left[\frac{a}{a_{0}}\left(\frac{a}{a_{0}}-1\right)\right]^{1 / 2}\right] \\
a_{+} & =\frac{a_{0}^{2}}{4}\left[\arccos \left(\frac{a}{a_{0}}\right)^{1 / 2}+\left(1-\frac{2 a}{a_{0}}\right)\left[\frac{a}{a_{0}}\left(1-\frac{a}{a_{0}}\right)\right]^{1 / 2}\right] \\
a_{+M} & =\frac{\pi}{8} a_{0}^{2}
\end{aligned}
$$

Choosing boundary conditions such that $\Phi_{\mathcal{E}}(0)=0$ (or $\alpha=\infty$ ), from eq. (157) one can explicitly write the discrete eigenvalues for the dust-dominated universe as

$$
\sqrt{\mathcal{E}}=\frac{2(4 n+1) \hbar}{a_{0}^{2}} \quad, \quad n=0,1, \ldots
$$

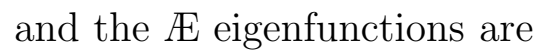

$$
\begin{aligned}
\Phi_{\mathcal{E}}[a] & =\frac{2^{\frac{3}{2}} e^{i \theta}}{\pi^{\frac{1}{2}} a_{0}}\left\{\cos \left[\frac{\sqrt{\mathcal{E}} a_{0}^{2}}{4 \hbar}\left[\arccos \left(\frac{a}{a_{0}}\right)^{\frac{1}{2}}+\left(1-\frac{2 a}{a_{0}}\right)\left[\frac{a}{a_{0}}\left(1-\frac{a}{a_{0}}\right)\right]^{\frac{1}{2}}\right]\right]\right. \\
& \left.-\sin \left[\frac{\sqrt{\mathcal{E}} a_{0}^{2}}{4 \hbar}\left[\arccos \left(\frac{a}{a_{0}}\right)^{\frac{1}{2}}+\left(1-\frac{2 a}{a_{0}}\right)\left[\frac{a}{a_{0}}\left(1-\frac{a}{a_{0}}\right)\right]^{\frac{1}{2}}\right]\right]\right\}
\end{aligned}
$$

for $a<a_{0}$ and

$$
\Phi_{\mathcal{E}}[a]=\frac{2^{\frac{3}{2}} e^{i \theta}}{\pi^{\frac{1}{2}} a_{0}} \exp \left\{\frac{\sqrt{\mathcal{E}} a_{0}^{2}}{4 \hbar}\left[\operatorname{arccosh}\left(\frac{a}{a_{0}}\right)^{\frac{1}{2}}+\left(1-\frac{2 a}{a_{0}}\right)\left[\frac{a}{a_{0}}\left(\frac{a}{a_{0}}-1\right)\right]^{\frac{1}{2}}\right]\right\}
$$

for $a>a_{0}$.

In the same way, one can solve for the stationary states of a radiation-dominated universe with $\gamma=4 / 3$. In this case the discrete eigenvalues are

$$
\sqrt{\mathcal{E}}=\frac{(4 n+1) \hbar}{a_{0}^{2}}
$$


and the $\mathbb{E}$-eigenfunctions are

$$
\begin{aligned}
\Phi_{\mathcal{E}}[a] & =\frac{2 e^{i \theta}}{\sqrt{\pi} a_{0}}\left\{\cos \left[\frac{\sqrt{\mathcal{E}} a_{0}^{2}}{2 \hbar}\left[\arccos \left(\frac{a}{a_{0}}\right)-\frac{a}{a_{0}}\left[1-\left(\frac{a}{a_{0}}\right)^{2}\right]^{1 / 2}\right]\right]\right. \\
& \left.-\sin \left[\frac{\sqrt{\mathcal{E}} a_{0}^{2}}{2 \hbar}\left[\arccos \left(\frac{a}{a_{0}}\right)-\frac{a}{a_{0}}\left[1-\left(\frac{a}{a_{0}}\right)^{2}\right]^{1 / 2}\right]\right]\right\}
\end{aligned}
$$

for $a<a_{0}$ and

$$
\Phi_{\mathcal{E}}[a]=\frac{2 e^{i \theta}}{\sqrt{\pi} a_{0}} \exp \left[\frac{\sqrt{\mathcal{E}} a_{0}^{2}}{2 \hbar}\left[\operatorname{arccosh}\left(\frac{a}{a_{0}}\right)-\frac{a}{a_{0}}\left[\left(\frac{a}{a_{0}}\right)^{2}-1\right]^{1 / 2}\right]\right]
$$

for $a>a_{0}$. The main properties of the wave function are essentially the same as those of the dust-dominated universe.

Note that in both the $\gamma>2 / 3$ and $\gamma<2 / 3$ cases, the $Æ$ eigenstates are real-valued. In the more conventional "Born-Oppenheimer" interpretation [16] of the Wheeler-de Witt equation, such wavefunctions represent a superposition of 'collapsing' and 'expanding' universes in the classically allowed region. In our formulation, by contrast, such states are stationary, and dynamics (such as the expansion and collapse of the universe) arises from their superposition. We will now illustrate such dynamics by

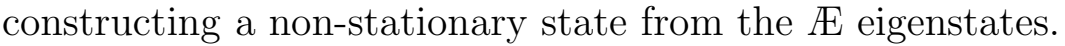

Let us consider the case of a $\gamma>2 / 3$ Friedman universe, which has a discrete spectrum for $\mathcal{E}$, and oscillating eigenfunctions in the classically allowed range $a \in\left[0, a_{0}\right]$. The general form of the wave packet for an arbitrary distribution $f\left[\rho_{n}\right]$ of "momenta" $\rho_{n} \doteq \sqrt{\mathcal{E}_{n}}$ is given by the standard formula

$$
\Psi[a, \tau]=\sum_{n=0}^{\infty} f\left[\rho_{n}\right] \Phi_{\rho_{n}}[a] e^{i \rho_{n}^{2} \tau / \hbar}
$$

where

$$
f\left[\rho_{n}\right]=\int_{0}^{\infty} d a \sqrt{|\mathcal{G}|} \Psi[a, 0] \Phi_{\rho_{n}}^{*}[a]
$$

and, for the choice of boundary conditions $\Phi_{\mathcal{E}}(0)=0$,

$$
\rho_{n}=\frac{\pi(4 n+1) \hbar}{4 a_{+M}}
$$

We build up the wave packet such that it is initially localized in the 'classically allowed' region $a \in\left[0, a_{0}\right]$ and it moves towards the singularity with "momentum" $\rho_{n}=\sqrt{\mathcal{E}_{n}}$ centered around $\rho_{n_{0}}$. For our example, we choose an initial wavepacket which is a triangle of unit height, with base of length $2 \delta$ centered at $a_{+0}$, i.e.

$$
\Psi\left[a_{+}, 0\right]=e^{i \rho_{n_{0}} a_{+} / \hbar}\left[1-\frac{\left|a_{+}-a_{+0}\right|}{\delta}\right]\left[\theta\left(a_{+}-a_{+0}+\delta\right)-\theta\left(a_{+}-a_{+0}-\delta\right)\right]
$$


where $\theta$ is the Heaviside step function.

Since the initial packet has support only in the region $a_{+}$, from eqs. (141), (166) and (168) we find

$$
\begin{aligned}
f\left[\rho_{n}\right] & =\int_{0}^{a_{+}} d a_{+} \Psi\left[a_{+}, 0\right] \Phi_{\rho_{n}}^{*}\left[a_{+}\right] \\
& =\frac{2 \hbar^{2}}{\delta}\left\{\frac { A ^ { * } } { ( \rho _ { n _ { 0 } } - \rho _ { n } ) ^ { 2 } } e ^ { i ( \rho _ { n _ { 0 } } - \rho _ { n } ) a _ { + 0 } / \hbar } \left[1-\cos \left[\left(\rho_{n_{0}}-\rho_{n}\right) \delta / \hbar\right]\right.\right. \\
& +\frac{B^{*}}{\left(\rho_{n_{0}}+\rho_{n}\right)^{2}} e^{i\left(\rho_{n_{0}}+\rho_{n}\right) a_{+0} / \hbar}\left[1-\cos \left[\left(\rho_{n_{0}}+\rho_{n}\right) \delta / \hbar\right]\right\}
\end{aligned}
$$

Inserting this result in eq. (165) and taking the modulus square, we finally find that the probability distribution of the wave packet evolves, in the classically allowed region $a_{+}$, as

$$
\begin{aligned}
\left|\Psi\left[a_{+}, \tau\right]\right|^{2} & =\frac{4 a_{+M}^{2}}{\pi^{4} \delta^{2}}\left\{\left[\sum_{n=0}^{\infty}\left[\cos \left(\mu_{+}(n)\right)+\sin \left(\sigma_{-}(n)\right)\right] \frac{\sin ^{2} \frac{\pi \delta\left(n-n_{0}\right)}{2 a_{+M}}}{\left(n-n_{0}\right)^{2}}\right.\right. \\
& \left.+\sum_{n=0}^{\infty}\left[\cos \left(\mu_{-}(n)\right)-\sin \left(\sigma_{+}(n)\right)\right] \frac{\sin ^{2} \frac{\pi \delta\left(n+n_{0}+1 / 2\right)}{2 a_{+}}}{\left(n+n_{0}+1 / 2\right)^{2}}\right]^{2} \\
& +\left[\sum_{n=0}^{\infty}\left[\sin \left(\mu_{+}(n)\right)-\cos \left(\sigma_{-}(n)\right)\right] \frac{\sin ^{2} \frac{\pi \delta\left(n-n_{0}\right)}{2 a_{+M}}}{\left(n-n_{0}\right)^{2}}\right. \\
& \left.\left.+\sum_{n=0}^{\infty}\left[\sin \left(\mu_{-}(n)\right)+\cos \left(\sigma_{+}(n)\right)\right] \frac{\sin ^{2} \frac{\pi \delta\left(n+n_{0}+1 / 2\right)}{2 a_{+}}}{\left(n+n_{0}+1 / 2\right)^{2}}\right]^{2}\right\}
\end{aligned}
$$

where, for convenience of notation, we have defined

$$
\mu_{ \pm}(n)=\frac{\pi}{a_{+M}}(n+1 / 4)\left[ \pm\left(a_{+}-a_{+0}\right)+\frac{\pi \hbar}{a_{+M}}(n+1 / 4) \tau\right]
$$

and

$$
\sigma_{ \pm}(n)=\frac{\pi}{a_{+M}}(n+1 / 4)\left[ \pm\left(a_{+}+a_{+0}\right)+\frac{\pi \hbar}{a_{+M}}(n+1 / 4) \tau\right]
$$

Similarly, the probability distribution in the classically forbidden region $a_{-}$is easily found to be

$$
\begin{aligned}
\left|\Psi\left[a_{-}, \tau\right]\right|^{2} & =\frac{4 a_{+M}^{2}}{\pi^{4} \delta^{2}} e^{\frac{\pi a_{-}}{2 a_{+}}}\left\{\left[\sum_{n=0}^{\infty}\left[\cos \left(\lambda_{1}(n)\right)+\sin \left(\lambda_{1}(n)\right)\right] \frac{\sin ^{2} \frac{\pi \delta\left(n-n_{0}\right)}{2 a_{+} M}}{\left(n-n_{0}\right)^{2}} e^{\frac{\pi a_{-}}{a_{+M}} n}\right.\right. \\
& \left.+\sum_{n=0}^{\infty}\left[\cos \left(\lambda_{2}(n)\right)-\sin \left(\lambda_{2}(n)\right)\right] \frac{\sin ^{2} \frac{\pi \delta\left(n+n_{0}+1 / 2\right)}{2 a_{+M}}}{\left(n+n_{0}+1 / 2\right)^{2}} e^{\frac{\pi a_{-}}{a_{+}} n}\right]^{2} \\
& +\left[\sum_{n=0}^{\infty}\left[\sin \left(\lambda_{1}(n)\right)-\cos \left(\lambda_{1}(n)\right)\right] \frac{\sin ^{2} \frac{\pi \delta\left(n-n_{0}\right)}{2 a_{+M}}}{\left(n-n_{0}\right)^{2}} e^{\frac{\pi a_{-}}{a} n} n\right. \\
& \left.+\sum_{n=0}^{\infty}\left[\sin \left(\lambda_{2}(n)\right)+\cos \left(\lambda_{2}(n)\right)\right] \frac{\sin ^{2} \frac{\pi \delta\left(n+n_{0}+1 / 2\right)}{2 a_{+M}}}{\left(n+n_{0}+1 / 2\right)^{2}} e^{\left.\frac{\pi a-M}{a_{+}} n\right]^{2}}\right\}
\end{aligned}
$$


where

$$
\lambda_{1}=\frac{\pi n}{a_{+M}}\left[\frac{\pi \hbar}{2 a_{+M}}(2 n+1) \tau-a_{+0}\right]
$$

and

$$
\lambda_{2}=\frac{\pi(2 n+1)}{2 a_{+M}}\left[\frac{\pi \hbar}{a_{+M}} n \tau+a_{+0}\right]
$$

For our illustrations, we have chosen to compute the probability distribution for a dust-dominated universe $(\gamma=1)$ with $n_{0}=400, a_{0}=1$ and $\delta=0.02$ (keeping $10^{4}$ terms in the series). The results are plotted in fig. 3, where different times are labeled by $\tau_{*} \doteq-10^{5} \tau$. For a comparison, we have also plotted in fig. 4 the modulus of the eigenfunction of $Æ$ for the $\mathcal{E}$ eigenvalue corresponding to the average $\mathcal{E}$ of the packet, namely $\mathcal{E}_{0}=(3202)^{2}$. This stationary state is the solution of a Wheeler-de Witt equation with a particular choice of operator-ordering and Planck's constant.

Fig. 3 shows the evolution of the wave packet; the packet begins by moving smoothly towards the singularity while gradually spreading. As it approaches the classical singularity at $a=0$, the packet starts oscillating, with the frequency and amplitude of oscillations increasing up to a maximum which is shown in more detail in fig. 5a. The wave packet is always zero at $a=0$, consistent with our choice of boundary conditions. As $\tau$ increases, the packet 'bounces' off $a=0$ and moves back towards the classical turning point at $a=a_{0}$. At the point of maximum expansion the wave packet has again an oscillating behaviour similar to that close to the classical singularity. Here, however, part of the wave packet extends into the 'classically forbidden' region $a>a_{0}$; there is a small, exponentially decaying probability to find the universe in such a region (see fig. 5b). At $a=a_{0}$ both the wave packet and its first derivative are continuous. As the time parameter continues to increase, the wave packet 'bounces' off $a=a_{0}$ and moves back towards the classical singularity. The packet rebounds repeatedly between $a=0$ and $a=a_{0}$ while gradually becoming delocalized. It is important to stress (see also [8]) that nothing pathological happens to the wave packet as it bounces between the classical singularity at $a=0$ and the turning point at $a=a_{0}$. In particular, there is no infinite compression of the wavefunction, and the transition from 'big crunch' to 'big bang' is a (relatively) smooth process, in this toy model, at the quantum level.

The norm of the wave packet has been numerically checked to be constant during all the stages of the evolution, as it obviously should.

Although we have specialized here to the case of a dust dominated universe, it is quite clear that we do not expect anything radically different from the analysis of other cases with $\gamma>2 / 3$. The same holds for the cases $\gamma<2 / 3$, the only difference being that there is only a single bounce of the kind shown in fig. 6, with the wave packet starting from infinite scale factor, 'bouncing' off the minimum radius $a_{0}$ and finally 
going back to infinite scale factor. Also, of course, the qualitative picture does not depend critically on the shape of this initial wavepacket.

As pointed out in section 2, the space of physical states is spanned by eigenstates of the evolution operator $\mathbb{E}$ with different $\mathcal{E}$, and in section 4 it was shown that this can be interpreted as leading to a quantum indeterminacy of Planck's constant according to formula (115). In our minisuperspace toy model, it is possible to directly relate the spread of the wavepacket in the scale factor $a$ to the spread in $\mathcal{E}$, and thereby to the dispersion in Planck's constant. Of course, this model is far too unrealistic to draw any quantitative conclusions regarding the dispersion of fundamental constants vs. the spread of the wavepacket in full quantum gravity.

To proceed, we construct a wave packet from eigenfunctions of $Æ$. Now, however, we want to introduce a more tractable Gaussian distribution in the momenta, and to simplify the analysis let us assume that the $\mathcal{E}$ eigenvalues are so closely spaced that we can replace sums over the discrete eigenvalues by an integral,|] i.e. we write

$$
\begin{aligned}
\Psi(a, \tau) & =\int_{-\infty}^{\infty} d \rho f[\rho] \Phi_{\rho}(a) e^{i \rho^{2} \tau / \hbar} \\
f(\rho) & =e^{-\beta^{2}\left(\rho-\rho_{0}\right)^{2}}
\end{aligned}
$$

where

$$
\sigma_{\rho}^{2} \doteq\left(2 \beta^{2}\right)^{-1}
$$

is the dispersion in the "momentum" $\rho=\sqrt{\mathcal{E}}$ distribution around the peak at $\rho_{0}$.

Evaluating the integral in the classically allowed region with $\Phi_{\rho}=e^{\frac{i \rho a_{+}}{\hbar}}$ and taking the modulus, we easily find that

$$
|\Psi[a, \tau]|^{2} \simeq \exp \left\{-\frac{1}{2} \frac{\left[a_{+}(a)+2 \sqrt{\mathcal{E}_{0}} \tau\right]^{2}}{\hbar^{2}\left[\beta^{2}+\frac{\tau^{2}}{\hbar^{2} \beta^{2}}\right]}\right\}
$$

where $a_{+}(a)$ is given by formula (138).

Following standard analysis (see, e.g., [17]), we see that, at any given $\tau$, the probability distribution of the scale factor $a$ is peaked around the set of semiclassical trajectories for which

$$
\left.a_{+}(a)\right|_{c l}=-2 \sqrt{\mathcal{E}_{0}} \tau
$$

or, in other words, for which the phase in the integral (176) is stationary (compare with eqs. (108) and (110)).

As an example, we can differentiate both sides of eq. (179) with respect to $\tau$ and find

$$
\frac{d a}{d \tau}=-\frac{2 \sqrt{\mathcal{E}_{0}}}{a}\left[\left(\frac{a}{a_{0}}\right)^{2-3 \gamma}-1\right]^{-1 / 2}
$$

\footnotetext{
${ }^{6}$ This can be done by replacing $\sum_{n=0}^{\infty} \rightarrow \frac{a_{+M}}{2 \pi \hbar} \int_{-\infty}^{\infty} d \rho$ for $\gamma>2 / 3$.
} 
If we now compare with the known solutions in classical cosmology for the perfect fluid matter content (see 13$]$ ), i.e.

$$
\frac{d a}{d t}=\left[\left(\frac{a}{a_{0}}\right)^{2-3 \gamma}-1\right]^{1 / 2}
$$

we can immediately infer the correspondence between the 'internal' time $t$ and the evolution parameter $\tau$ at the classical level, given by

$$
d \tau=-\frac{a}{2 \sqrt{\mathcal{E}_{0}}}\left[\left(\frac{a}{a_{0}}\right)^{2-3 \gamma}-1\right] d t
$$

We now expand $|\Psi[a, \tau]|^{2}$ in eq. (178) around the classical trajectories with $a=$ $a\left(\left.a_{+}\right|_{c l}\right)+\Delta a$ at $\tau$ fixed, and find

$$
|\Psi[a, \tau]|^{2} \simeq e^{-\frac{\left(a-a_{c l}\right)^{2}}{2 \sigma_{a}^{2}}}
$$

where

$$
\sigma_{a}^{2}(\beta, \tau)=\left[\beta^{2}+\frac{\tau^{2}}{\hbar^{2} \beta^{2}}\right]\left[\frac{\partial a_{+}}{\partial a}\right]^{-2}\left(\frac{2 \hbar G}{3 \pi}\right)^{2}
$$

and we have reinserted dimensions according to

$$
a \rightarrow \sqrt{\frac{2 G}{3 \pi}} a
$$

Eq. (183) can be interpreted as saying that the probability to find the universe in a certain configuration with scale factor $a$ at time $\tau$ is given by a Gaussian peaked at the classical trajectory $a_{c l}$ with a dispersion $\sigma_{a}^{2}$.

Finally, as an exercise, we can tentatively give a lower bound on the dispersion for the scale factor by minimizing it with respect to $\beta$, and we find

$$
\left\{\operatorname{Min}\left[\sigma_{a}^{2}\right]\right\}_{\beta=|\tau / \hbar|^{1 / 2}}=\left|a_{+}\right|\left[\frac{\partial a_{+}}{\partial a}\right]^{-2} L_{P, e f f}^{2}
$$

where we have defined $L_{P, e f f}^{2}=\frac{2 \hbar_{e f f} G}{3 \pi}$ and the 'dressed' Planck's constant $\hbar_{e f f}$ is introduced according to eq. (115). We can study the behaviour of eq. (186) for some simple examples of matter content in the universe. In the case of a de Sitter universe with $\gamma=0$ we can use formula (153) for $a_{+}$, define $a=\xi a_{0}(\xi \geq 1)$ and find

$$
\frac{\left\{\operatorname{Min}\left[\sigma_{a}^{2}\right]\right\}_{\beta=|\tau / \hbar|^{1 / 2}}}{L_{P, \text { eff }}^{2}}=\frac{\left(\xi^{2}-1\right)^{1 / 2}}{3 \xi^{2}}<\frac{1}{36} \quad, \quad \forall \xi \geq 1
$$

Therefore, if the dispersion in the scale factor is such to be minimized with respect to $\beta$, it will always be negligible for the case $\gamma=0$. A similar conclusion can be easily 
drawn by studying the case of a radiation-dominated universe. Unfortunately, this simple analysis does not either provide any reliable upper bound on $\sigma_{a}$.

For a dust-dominated universe, instead, using eq. (158) with $a=\xi a_{0}(\xi \leq 1)$, one has

$$
\frac{\left\{\operatorname{Min}\left[\sigma_{a}^{2}\right]\right\}_{\beta=|\tau / \hbar|^{1 / 2}}}{L_{P, \text { eff }}^{2}}=\frac{\left[\arccos (\xi)^{1 / 2}+(1-2 \xi)[\xi(1-\xi)]^{1 / 2}\right]}{4 \xi(1-\xi)} \rightarrow \infty \quad, \quad \xi \rightarrow 0
$$

In this toy model, it is therefore possible to have non-negligible dispersion close to the classical singularity, while having $\sigma_{a}$ negligible at much later times.

\section{Conclusions}

In this article we have pointed out that there is a constant (denoted $\mathcal{E}$ ) in the classical Hamiltonian of certain parametrized theories, which cannot be determined from the classical trajectories. It is the arbitrariness of this constant in classical physics which allows us to extend the space of physical states in the corresponding quantum theory, and this is the key to our proposed resolution of the problem of time. Solutions of the Wheeler-de Witt equations are stationary states, in our scheme, but there is an infinite set of such equations, each with its own parameter $\mathcal{E}$. Non-stationary states are composed of superpositions of such states, with different $\mathcal{E}$ parameters. It was seen that this formalism can be derived directly from a transfer-matrix quantization of parametrized theories.

As noted in section 4 , the constant $\mathcal{E}$ in the Wheeler-de Witt equation (equivalent to $Æ \Phi_{\mathcal{E}}=-\mathcal{E} \Phi_{\mathcal{E}}$ in our notation) can always be absorbed into a redefinition of Planck's constant $\hbar_{e f f}=\hbar / \sqrt{\mathcal{E}}$. In the special case of pure gravity, the constant $\mathcal{E}$ could alternatively be absorbed into a redefinition of Newton's constant (45) (as was done in ref. (4), but in pure gravity this is anyway equivalent to rescaling the Planck constant, since only the combination $\hbar G / \sqrt{\mathcal{E}}$ appears in the Wheeler-de Witt equation in this case. In the general case, any non-stationary state

$$
\Psi[q, \tau]=\sum_{\mathcal{E}} a_{\mathcal{E}} \Phi_{\mathcal{E}}[q] e^{i \mathcal{E} \tau / \hbar}
$$

involves a superposition of Wheeler-de Witt wavefunctions $\Phi_{\mathcal{E}}[q]$, each with a different effective value of Planck's constant, so in this sense any non-stationary physical state entails an inherent uncertainty in the value of Planck's constant. Unfortunately, we have not been able to place a reliable lower bound on this uncertainty, for reasons which were discussed in section 4 , above. Nevertheless, the possibility that there is an inherent uncertainty in the effective value of Planck's constant, which might be large 
enough to be observable, raises some interesting phenomenological questions, which we hope to address in the near future.

\section{Acknowledgements}

It is a pleasure to acknowledge the hospitality of the Lawrence Berkeley Laboratory, where most of this work was carried out. A.C. was supported by an INFN grant at the Lawrence Berkeley Laboratory. J.G.'s research is supported in part by the U.S. Dept. of Energy, under Grant No. DE-FG03-92ER40711.

\section{A Appendix}

We briefly comment here on a 2-d minisuperspace model for a universe filled with a homogeneous scalar field $\phi$ with arbitrary potential $\mathcal{V}(\phi)$ and with a bare cosmological constant $\lambda$. The geometrical ansatz is the same as that of the 1-d minisuperspace model, i.e. the isotropic and homogeneous Friedmann metric given by eq. (129). The reparametrization invariant action for such a model is

$$
S_{s c}=\frac{1}{2} \int d t\left[-\frac{a \dot{a}^{2}}{N}+\frac{a^{3} \dot{\phi}^{2}}{N}+N a-N(\lambda+\mathcal{V}(\phi)) a^{3}\right]
$$

This can be easily written in the Hamiltonian form of eq. (50), with $p_{a}=-\frac{a \dot{a}}{N}, p_{\phi}=\frac{a^{3} \dot{\phi}}{N}$, $m=1$ and

$$
\begin{aligned}
G_{a a} & =-a \\
G_{\phi \phi} & =a^{3} \\
V(a, \phi) & =\frac{a}{2}\left[(\mathcal{V}(\phi)+\lambda) a^{2}-1\right]
\end{aligned}
$$

The general form of the evolution operator $Æ$ is

$$
Æ=-\frac{3 \hbar^{2}}{2|\mathcal{G}|^{1 / 2}}\left[\frac{\partial}{\partial a}|\mathcal{G}|^{1 / 2} \mathcal{G}^{a a} \frac{\partial}{\partial a}+\frac{\partial}{\partial \phi}|\mathcal{G}|^{1 / 2} \mathcal{G}^{\phi \phi} \frac{\partial}{\partial \phi}\right]+\frac{\hbar^{2} \mathcal{R}}{2}
$$

where, as usual, we have introduced the 'supermetric' $\mathcal{G}_{m n}=2 V G_{m n}$, with determinant $\mathcal{G}$ and corresponding scalar curvature

$$
\mathcal{R}=\frac{1}{2 a V}\left[\left(1+\frac{2 V}{a}\right) \frac{1}{V^{2}}+\frac{2}{a^{2}} \frac{\partial^{2}}{\partial \phi^{2}} \ln V\right]
$$


As a consequence of the hermiticity requirements for the measure, similarly to the 1-d minisuperspace case, the formal expression of the evolution operator is not well defined at the 'turning points' where $V=0$. In fact, from eqs. (191)-(193) and using the explicit form of the supermetric, one gets

$$
\begin{aligned}
\rightleftarrows & =\frac{3 \hbar^{2}}{4 a V}\left\{\frac{\partial^{2}}{\partial a^{2}}+\frac{1}{a} \frac{\partial}{\partial a}-\frac{1}{a^{2}} \frac{\partial^{2}}{\partial \phi^{2}}+2 \delta(V) \operatorname{sign}(V)\left[\frac{\partial V}{\partial a} \frac{\partial}{\partial a}\right.\right. \\
& \left.\left.-\frac{1}{a^{2}} \frac{\partial V}{\partial \phi} \frac{\partial}{\partial \phi}\right]+\frac{1}{3}\left[\left(1+\frac{2 V}{a}\right) \frac{1}{V^{2}}+\frac{2}{a^{2}} \frac{\partial^{2}}{\partial \phi^{2}} \ln V\right]\right\}
\end{aligned}
$$

The general procedure will be to study the eigenvalue problem for $\mathbb{E}$ (eq. (136)) in the two regions where $V>0$ and $V<0$ separately, and then impose suitable joining conditions on the partial derivatives of the eigenfunctions at the 'turning point' $V=0$.

The simplest problem which we can analyze is that corresponding to a massless and minimally coupled scalar field[ with

$$
\mathcal{V}(\phi)=\lambda=0
$$

for which $V=-\frac{a}{2}$ and $\mathcal{R}=0$. In this case, since the scale factor $a$ is constrained to be positive, the evolution operator given by eq. (194) has no ambiguities due to delta terms and becomes

$$
Æ=-\frac{3 \hbar^{2}}{2 a^{2}}\left[\frac{\partial^{2}}{\partial a^{2}}+\frac{1}{a} \frac{\partial}{\partial a}-\frac{1}{a^{2}} \frac{\partial^{2}}{\partial \phi^{2}}\right]
$$

The hyperbolic problem described by eq. (136) with the operator (196) and with $\mathcal{E}=1$ has been extensively discussed in the literature as an ordinary differential problem (see, e.g., 18 and references therein) or as a Klein-Gordon eigenvalue problem with respect to a peculiar measure (see, e.g., [20]). One can separate the differential equation and express bounded solutions as a superposition of modified Bessel functions of imaginary order 22

$$
\Psi_{\mathcal{E}, k}[a, \phi]=\exp ( \pm i k \phi / \hbar) K_{\frac{i k}{2 \hbar}}\left(\frac{\sqrt{\mathcal{E}} a^{2}}{\sqrt{6} \hbar}\right)
$$

where $k$ is the separation constant, or give them as a product of harmonic oscillator wave functions like

$$
\Psi_{\mathcal{E}, n}[a, \phi]=H_{n}\left[\left(\frac{2 \mathcal{E}}{3 \hbar^{2}}\right)^{1 / 4} X\right] H_{n}\left[\left(\frac{2 \mathcal{E}}{3 \hbar^{2}}\right)^{1 / 4} Y\right] e^{-\sqrt{\frac{\mathcal{E}}{6} \frac{\left(X^{2}+Y^{2}\right)}{\hbar}}}
$$

where $X=a \cosh \phi$ and $Y=a \sinh \phi[18$.

Unfortunately, neither of these solutions are orthogonal with respect to the measure $\sqrt{\mathcal{G}} \propto a^{3}$, as it is easy to check. To guarantee the self-adjointness of the evolution

\footnotetext{
${ }^{7}$ The case of a conformally coupled scalar field turns out to be essentially equivalent.
} 
operator, one has to construct a particular superposition of any of such solutions which satisfies appropriate conditions on the boundary of the 2-d minisuperspace.

A possible method of solution of the eigenvalue problem makes use of the so called 'Rindler' coordinates 21]. Here we derive the formal boundary conditions for the eigenfunctions required for Hermiticity, even though we have not been able to explicitly solve for them. The idea is to make the following transformation of coordinates in the 2-d minisuperspace :

$$
\begin{aligned}
& x=\frac{a^{2}}{\sqrt{6}} \cosh 2 \phi \\
& y=\frac{a^{2}}{\sqrt{6}} \sinh 2 \phi
\end{aligned}
$$

The minisuperspace line element in the new coordinates takes the simple form $d s_{\text {Super }}^{2}=\left(d y^{2}-d x^{2}\right) / 4$. The minisuperspace region $a \in[0, \infty)$ and $\phi \in(-\infty, \infty)$ is mapped into the right Rindler wedge of a 2-d Minkowski spacetime, with boundary $|x|<y$. The correspondence between old and new coordinates is one-to-one, with $a=0$ mapped to $x= \pm y, \phi=$ constant to $\frac{x}{y}=$ constant lines and $a=$ constant mapped to the hyperbolae $x^{2}-y^{2}=$ constant. The evolution operator simply becomes the 2 -d wave operator in the Rindler wedge, i.e.

$$
\rightleftarrows=\hbar^{2}\left(\frac{\partial^{2}}{\partial y^{2}}-\frac{\partial^{2}}{\partial x^{2}}\right)
$$

By further 'tilting' the minisuperspace axes by $45^{\circ}$ with the coordinates

$$
\begin{aligned}
& x_{+}=\frac{1}{2}(x+y) \\
& x_{-}=\frac{1}{2}(x-y)
\end{aligned}
$$

$\left(x_{ \pm} \in[0, \infty)\right)$, the evolution operator becomes

$$
\rightleftarrows=-\hbar^{2} \frac{\partial^{2}}{\partial x_{+} \partial x_{-}}
$$

with constant measure. The general solution of the differential equation (136) is thus a linear combination of plane waves

$$
\Phi_{\mathcal{E}}\left[x_{+}, x_{-}\right]=\int d \lambda\left[A(\lambda) e^{i\left(\lambda x_{+}-\frac{\mathcal{E}}{\lambda} x_{-}\right) / \hbar}+B(\lambda) e^{i\left(\lambda x_{-}-\frac{\mathcal{E}}{\lambda} x_{+}\right) / \hbar}\right]
$$

Moreover, the self-adjointness condition for the evolution operator $Æ$ requires that

$$
\int_{0}^{\infty} d x_{+} \int_{0}^{\infty} d x_{-} \Phi_{\mathcal{E}_{1}}^{*} \frac{\partial^{2}}{\partial x_{+} \partial x_{-}} \Phi_{\mathcal{E}_{2}}=\int_{0}^{\infty} d x_{+} \int_{0}^{\infty} d x_{-} \Phi_{\mathcal{E}_{2}} \frac{\partial^{2}}{\partial x_{+} \partial x_{-}} \Phi_{\mathcal{E}_{1}}^{*}
$$


Integrating by parts, one can see that this is true if the following boundary condition for the eigenfunctions holds

$$
\int_{0}^{\infty} d x_{-}\left[\Phi_{\mathcal{E}_{1}}^{*} \frac{\partial}{\partial x_{-}} \Phi_{\mathcal{E}_{2}}\right]_{x_{+}=0}^{x_{+}=\infty}=\int_{0}^{\infty} d x_{+}\left[\Phi_{\mathcal{E}_{2}} \frac{\partial}{\partial x_{+}} \Phi_{\mathcal{E}_{1}}^{*}\right]_{x_{-}=0}^{x_{-}=\infty}
$$

Alternatively, one can transform the 'infinite' boundary to a finite one by use of the following conformal transformation on the superspace metric $d s_{\text {Super }}^{2}$ :

$$
\begin{aligned}
d \tilde{s}_{\text {Super }}^{2} & =\Omega^{2} d s_{\text {Super }}^{2}=-d u d v & & \\
u & =2 \arctan [(x-y)], & & u \in[0, \pi] \\
v & =2 \arctan [(x+y)], & & v \in[0, \pi] \\
\Omega^{2} & =16 \cos ^{2}\left(\frac{u}{2}\right) \cos ^{2}\left(\frac{v}{2}\right) & &
\end{aligned}
$$

In this case the evolution operator transforms as

$$
Æ=-16 \hbar^{2} \cos ^{2}\left(\frac{u}{2}\right) \cos ^{2}\left(\frac{v}{2}\right) \frac{\partial^{2}}{\partial u \partial v}
$$

with constant measure, and it is self-adjoint if

$$
\int_{0}^{\pi} d u\left[\Phi_{\mathcal{E}_{1}}^{*} \frac{\partial}{\partial u} \Phi_{\mathcal{E}_{2}}\right]_{v=0}^{v=\pi}=\int_{0}^{\pi} d v\left[\Phi_{\mathcal{E}_{2}} \frac{\partial}{\partial v} \Phi_{\mathcal{E}_{1}}^{*}\right]_{u=0}^{u=\pi}
$$

The eigenfunctions are the same as those given in eq. (203), provided one makes the substitutions $x_{+} \rightarrow 2 \tan \frac{v}{2}, x_{-} \rightarrow 2 \tan \frac{u}{2}$ and $\mathcal{E} \rightarrow \mathcal{E} / 16$.

Thus, formally, the hyperbolic eigenvalue problem of eq. (136) for the 2-d minisuperspace model with the minimally coupled scalar is solved provided one fixes the coefficients $A$ and $B$ in the expansion (203) such that to satisfy given Cauchy data and the condition (205) (or (208)) at the boundary. In particular, although we cannot give an explicit formula, the problem (208) certainly admits a solution. A possible simple example would be, for instance, a wave function such that $\Phi_{\mathcal{E}}(u, 0)\left(\Phi_{\mathcal{E}}(0, v)\right)$ has a fixed parity with respect to the point $u=\frac{\pi}{2}\left(v=\frac{\pi}{2}\right)$. 


\section{References}

[1] K. Kuchar, in Quantum Gravity 2: A second Oxford symposium, ed. C. Isham, R. Penrose, and D. Sciama (Oxford University Press, Oxford, 1981).

[2] K. Kuchar, Time and interpretations of quantum gravity, Proceedings of the 4th Canadian Conference on General Relativity and Astrophysics, ed. G. Kunstatter et. al. (World Scientific, Singapore, 1992).

[3] C. J. Isham, Imperial College preprint IMPERIAL-TP-91-92-25, bulletin board: gr-qc/9210011.

[4] J. Greensite, Phys. Rev. D49 (1994) 930.

[5] U. Gerlach, Phys. Rev. 177 (1969) 1929.

[6] C. W. Misner, K. S. Thorne, and J. A. Wheeler, Gravitation (W.H. Freeman and Co., New York, 1973).

[7] N. C. Tsamis and R. P. Woodard, Phys. Rev. D36 (1987) 3641.

[8] B. S. de Witt, Phys. Rev. 160 (1968) 1113.

[9] V. Moncrief and C. Teitelboim, Phys. Rev. D6 (1972) 966.

[10] M. Ryan, Hamiltonian Cosmology (Springer-Verlag, Berlin, 1972).

[11] Y. Peleg, The wave function of a collapsing star and quantization conditions, hep-th@xxx/9303169.

[12] B. F. Schutz, Phys. Rev. D4 (1971) 3559.

[13] G. F. R. Ellis, in General Relativity and Cosmology, XLVIII E. Fermi Summer School (ed. R.K. Sachs, Academic, New York, 1971).

[14] M. Reed and B. Simon, Methods of Modern Mathematical Physics: Functional Analysis (Academic, New York, 1972).

[15] J. B. Hartle and S. W. Hawking, Phys. Rev. D28 (1983) 2960.

[16] T. Banks, Nucl. Phys. B249 (1985) 332; J.J. Halliwell and S.W. Hawking, Phys. Rev. D31 (1985) 1777; A. Vilenkin, Phys. Rev. D39 (1989) 1116, R. Brout and G. Venturi, Phys. Rev. D39 (1989) 2436.

[17] A. Messiah, Quantum Mechanics (North Holland, Amsterdam, 1962). 
[18] S. W. Hawking and D. N. Page, Phys. Rev. D42 (1990) 2655.

[19] D. N. Page, J. Math. Phys. 32 (1991) 3427.

[20] S. A. Fulling, Phys. Rev. D7 (1973) 2850.

[21] W. Rindler, Am. J. Phys. 34 (1966) 1174.

[22] M. Abramowitz and I. A. Stegun, Handbook of Mathematical Functions (Dover, New York, 1965). 


\section{Figure Captions}

Fig. 1 Classical evolution via $\partial_{\tau} Q=\{Q, \mathbb{E}\}$. The final configuration $q^{\prime}, q^{\prime \prime}, \ldots$ depends on the $N, N_{i}$ functions, but the classical action $S^{\mathcal{E}}\left[q_{f}, q_{0}\right]$ from the initial to final configurations is proportional to the evolution parameter $\tau$, independent of $N, N_{i}$.

Fig. 2 Expansion of the (semiclassical) wavepacket. WKB wavefunctions $\Psi[q, \tau]$ are peaked, at any parameter time $\tau$, on a set of configurations $\left\{q_{c l}\right\}$ corresponding to hypersurfaces of the classical 4-manifold, such that $\tau=-S^{\mathcal{E}}\left[q_{0}, q_{c l}\right] / 2 \mathcal{E}$.

Fig. 3 Evolution of a 'triangular' wavepacket for a dust dominated universe. The parameters chosen are $n_{0}=400, a_{0}=1, \delta=0.02$ and different times are labeled by $\tau_{*}=-10^{5} \tau$.

Fig. 4 The modulus of the eigenfunction of $Æ$ for the average eigenvalue $\mathcal{E}_{0}=(3202)^{2}$ of the wave packet.

Fig. 5 a) The wave packet at its closest approach to the classical singularity.

b) The wave packet at the radius of maximum classical expansion. 
This figure "fig1-1.png" is available in "png" format from: http://arxiv.org/ps/gr-qc/9406044v1 


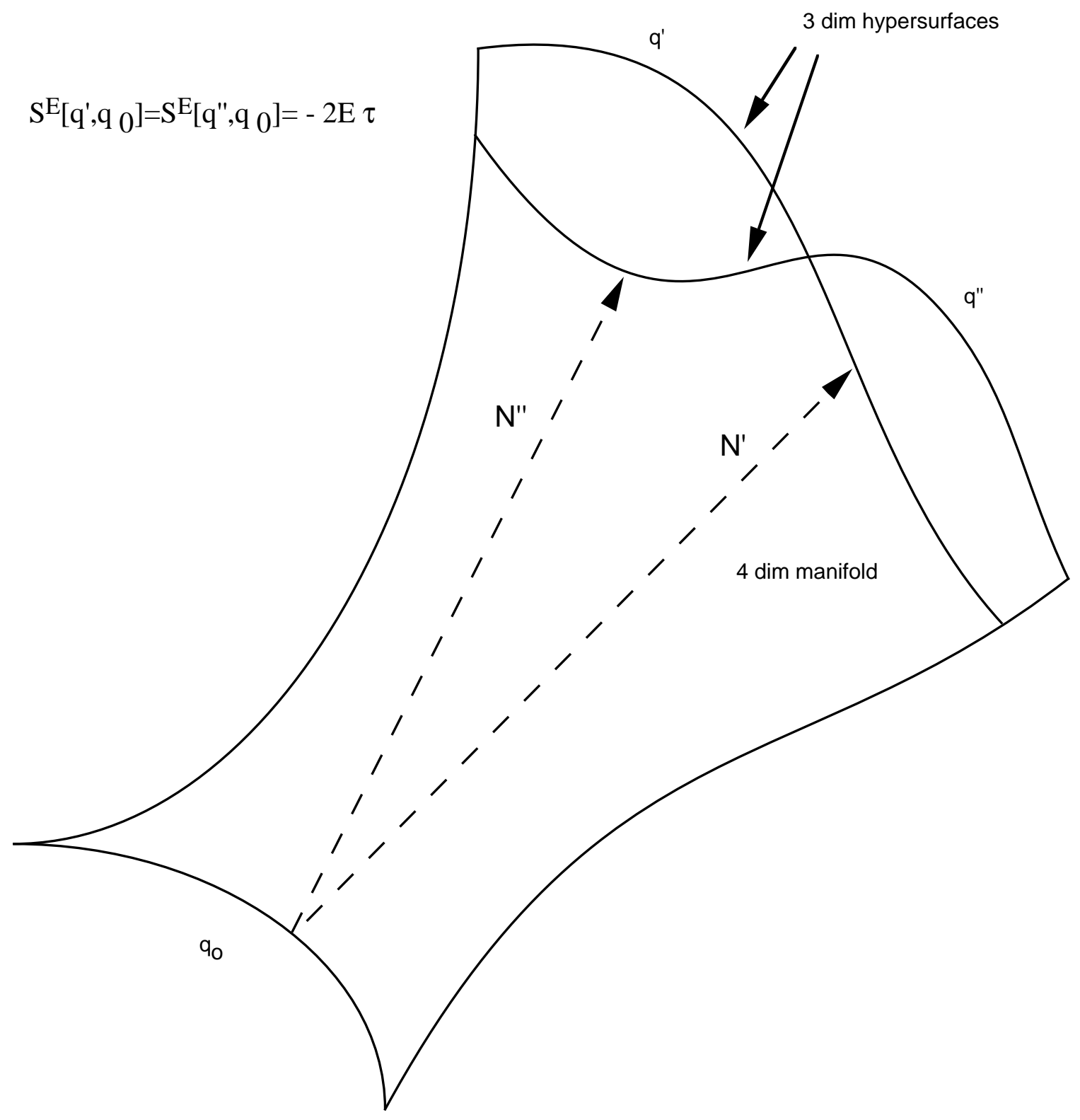


This figure "fig2-1.png" is available in "png" format from: http://arxiv.org/ps/gr-qc/9406044v1 
This figure "fig1-2.png" is available in "png" format from: http://arxiv.org/ps/gr-qc/9406044v1 
This figure "fig2-2.png" is available in "png" format from: http://arxiv.org/ps/gr-qc/9406044v1 


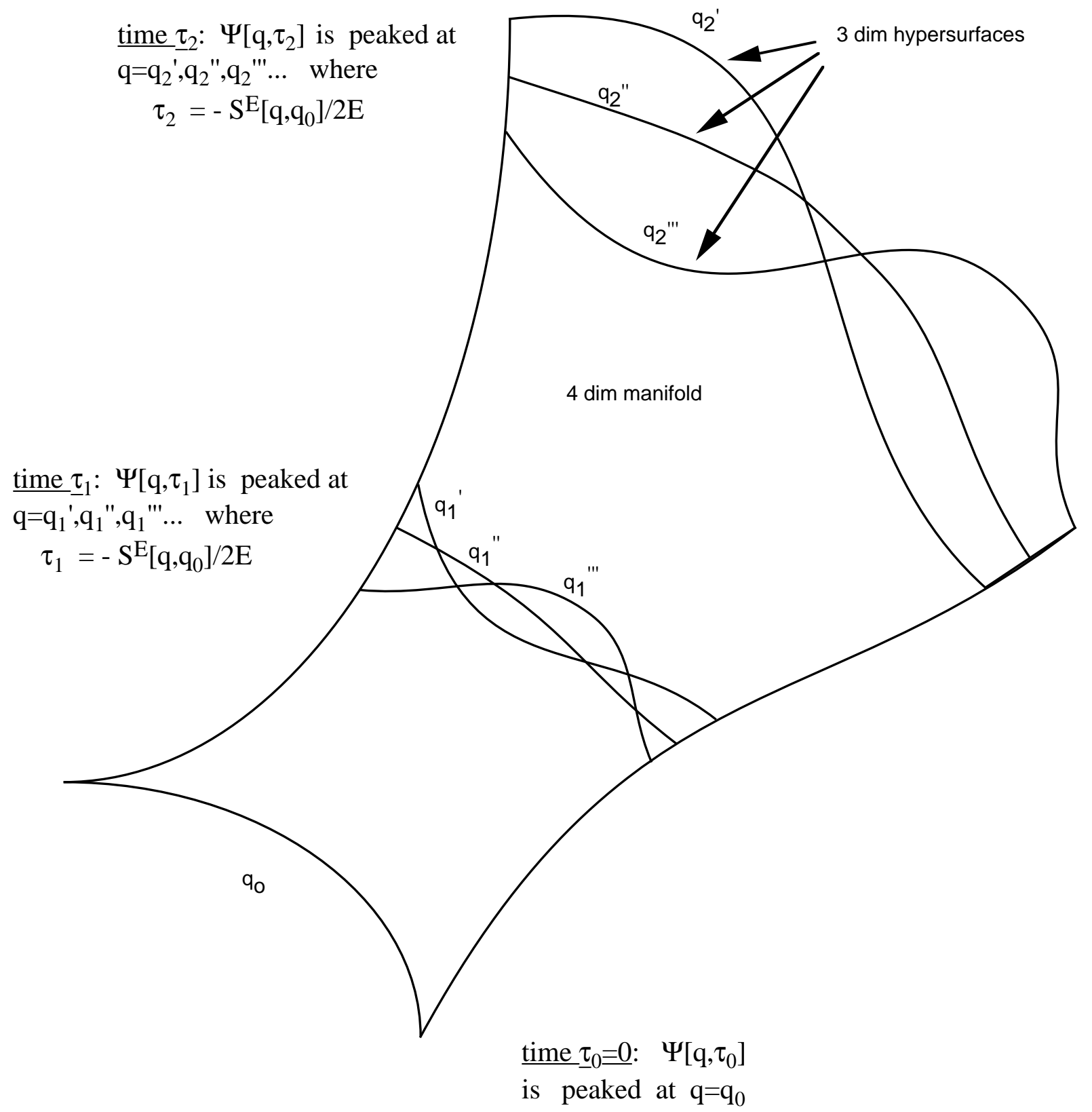


This figure "fig1-3.png" is available in "png" format from: http://arxiv.org/ps/gr-qc/9406044v1 


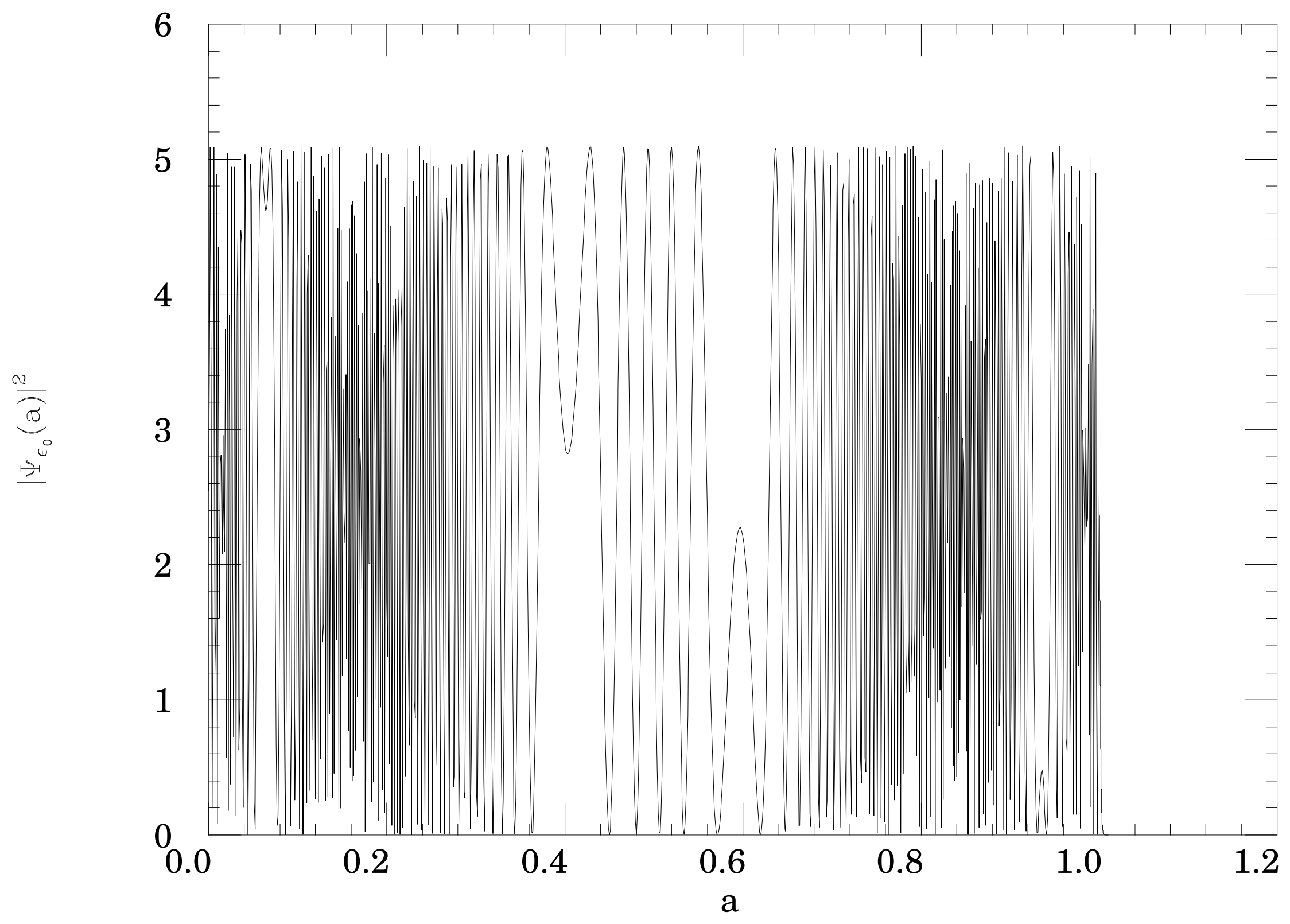



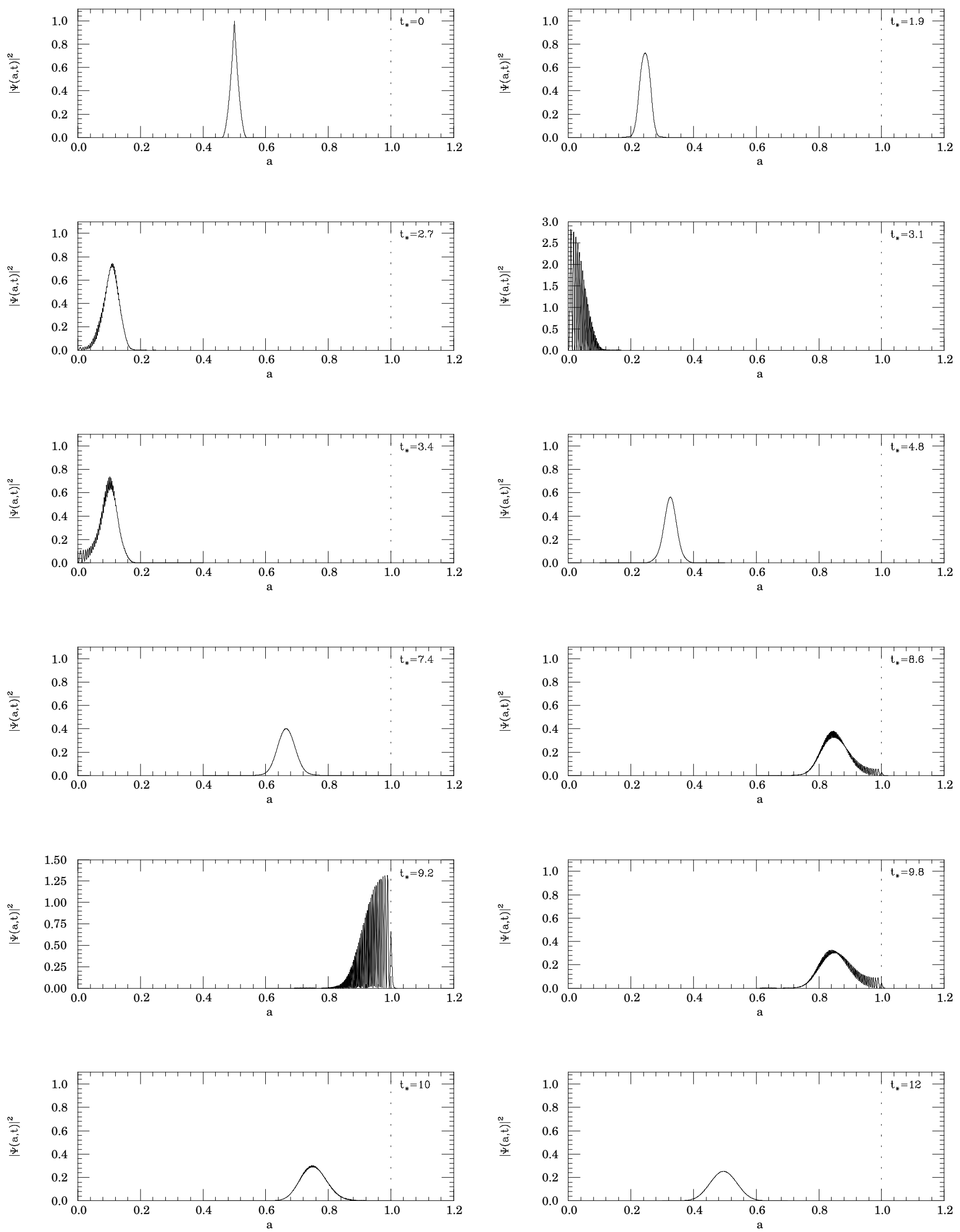

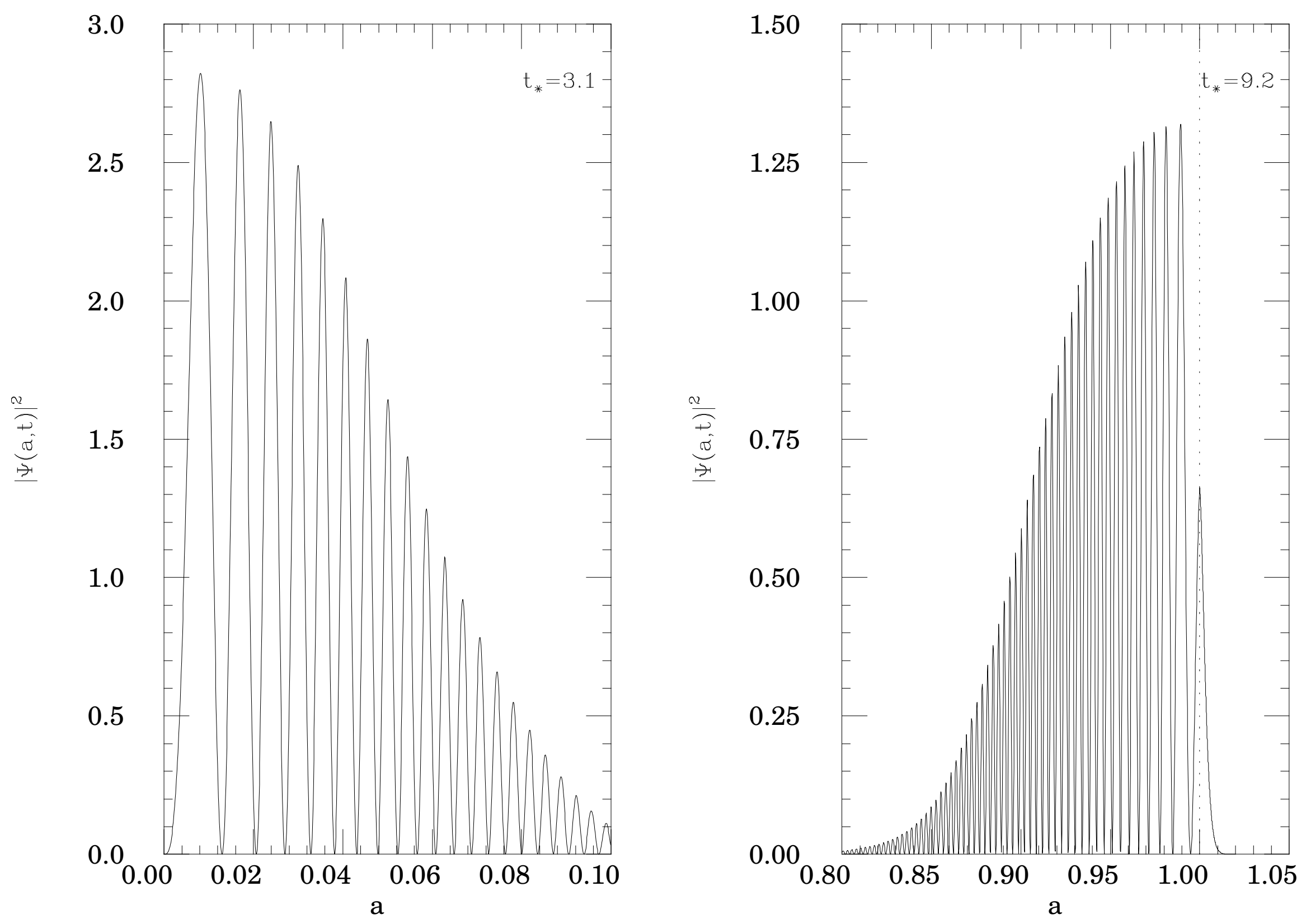